\title{
Effect of geometry and fill level on the transport and mixing behaviour of a co-rotating twin screw extruder
}

\author{
Martin Robinson $^{\mathrm{a}}$ and Paul W. Cleary ${ }^{\mathrm{b}}$ \\ ${ }^{a}$ Department of Computer Science, University of Oxford, Wolfson Building, Parks Road, \\ Oxford, OX1 3QD, United Kingdom \\ ${ }^{\mathrm{b}}$ CSIRO Data61, Clayton, Australia \\ * Corresponding author: paul.cleary@csiro.au
}

\begin{abstract}
The transport and mixing properties of a co-rotating twin screw extruder with both screw elements and kneading blocks are explored using the Smoothed Particle Hydrodynamics (SPH) method. The use of a Lagrangian method enables simulation of extruders partially or fully filled with fluid and is not limited by problems associated with extreme mesh deformation due to the opposite rotation of the impellors or the small gaps between them. The cross-channel and axial fluid transport and mixing in the screw elements and kneading blocks are analysed. The effect of parametric variations in both impeller elements on the nature of the fluid flow and the mixing are explored; specifically the effects of varying the screw pitch (positively correlated to mixing rate), kneading block stagger angle (the mixing rate peaks at a block angle of 30 degrees) and gap size between the rotating elements (positively correlated to mixing rate). Flow and mixing behaviour is also investigated for partially filled cases, with a fill level of $50 \%$ producing the highest mixing rates for the screw elements. The presence of fluid free surfaces are shown as generally positive for mixing. We demonstrate significantly improved mixing when the orientation handedness of the screw and kneading blocks leads to concentration of fluid in the kneading elements.
\end{abstract}




\section{Keywords}

SPH, mixing, extrusion, screw, kneading, FTLE 


\section{Introduction}

Twin screw extruders are popular devices in the food, polymer and other industries where the mixing of highly viscous fluids is required. While their use is widespread in industry, there is still a lack of understanding of the flow patterns and their effect on the mixing and fluid transport properties of the extruder. This restricts improvement of existing processes, optimisation of extruder design and the development of new products.

The methodology used in this paper involves prediction of the flow field and then analysis of mixing behaviour and was presented in Robinson et al. (2008), where two dimensional (2D) simulations of a Twin Cam mixer were successfully validated against published FEM and experimental results. Robinson and Cleary (2011) then analysed mixing processes in a similar Twin Cam mixer and explored the effects of variations in impeller geometry and operating conditions. This paper uses the methodology developed in these papers for a more realistic three dimensional (3D) geometry, namely the Twin Screw Extruder.

In recent years there has been an increasing emphasis on the CFD modelling of Twin Screw Extruders (Connelly and Kokini, 2007; Rios et al. 1998; Cheng and Manas-Zloczower, 1998; Ishikawa et al. 2001, 2002; Kajiwara et al. 1996; Lawal and Kalyon 1995; Valette et al. 2008). These simulations provide a more detailed insight into the flow than is possible with experimental studies, and allow greater flexibility and reduced setup time in varying the geometry or the operational parameters.

The Finite Element Method (FEM) is the numerical technique most often applied to such systems. Connelly and Kokini (2007) and Bertrand et al. (2003) simulated a 2D crosssection of a Twin Screw Extruder. At low Reynolds numbers, the 2D assumption is valid 
for extruders containing only screw elements. However, the inclusion of kneading blocks creates a fully 3D flow field. Cheng and Manas-Zloczower (1998), Ishikawa et al. (2001), Kajiwara et al., Lawal and Kalyon (1995), Valette et. al. (2008) and Sobhani et. al. (2013) all performed 3D simulations of a Twin Screw Extruder using FEM, simulating individual screw or kneading elements in isolation. Hétu and Ilinca (2013) used FEM and an immersed boundary method to simulate the combination of screw and mixing elements, but did not consider kneading blocks. In this paper we consider both screw and kneading elements and their combination, allowing the interaction between them to be investigated.

The Smoothed Particle Hydrodynamics (SPH) method of Monaghan (1994, 2005) is here used to simulate flow and mixing in the Twin Screw Extruder. SPH is a Lagrangian particle method for simulating fluid flow. Rather than using a fixed grid, the fluid variables are interpolated over particles which move with the fluid velocity. Each particle represents a volume of fluid, so it can have its own physical properties, and the advection is obtained automatically from the motion of the particles. SPH has proved to be a useful method for modelling many different classes of industrial applications. For example, Cleary et al. (2007) discusses the use of SPH in die casting, resin transfer moulding, pyrometallurgical processes with reactive solids, slurry flow in sag mills and the mixing of large particulate solids, Yakutovich et. al. (2009) use SPH to model liquid crystal displays and Kulasegaram et. al. (2011) use it to model self-compacting concrete. SPH modelling of fluid structure interaction for coastal and offshore waves is particularly popular, for example papers by Veen and Gourlay (2012), Altomare et. al. (2014) and Gómez-Gesteira and Dalrymple (2004). Previously, the authors have applied SPH to study mixing processes in batch helical ribbon mixers (Robinson and Cleary 2012), 2D twin cam mixers (Robinson and Cleary 2008, 2011) and preliminary analysis of Twin Screw Extruders (Cleary and Robinson, 
2011). More recently, Eitzlmayr and Khinast (2015a\&b) used SPH combined with an analytic wall model for approximately narrow boundary regions of the flow to study flow and mixing in the Twin Screw Extruder containing only screw elements.

The primary advantages of using the SPH method over FEM for this application is that SPH naturally handles the use of complex, moving geometries and fluid free-surfaces. The geometry of the Twin Screw Extruder contains a pair of intermeshing and rotating screws or kneading blocks within a stationary barrel-shaped outer channel. For an accurate FEM simulation, this would normally require re-meshing at every timestep, or the use of a method such as the Lagrange multiplier based fictitious domain method (Bertrand et al. 2003). Instead, SPH boundaries are constructed from particles following the approach of Monaghan (2003). Moving components such as impellors can be included in a simulation by assigning the appropriate velocity to each boundary particle at each time based on its rigid body motion.

Unlike Eulerian methods, there is no need to explicitly track the fluid surface, which is implicitly defined by the SPH particle positions. Therefore, any changes over time to the topology of the free-surface (e.g. the merging or separation of fluid volumes) are all naturally handled by the method. A Twin Screw Extruder is normally operated in starvedfeeding conditions (Van Der Goot et al. 1998), where the feed rate of fluid into the extruder is set so that the initial screw sections of the device are only partially filled. This creates a free-surface with a shape determined by the ratio of gravity and viscous forces (de Graaf et al. 1999). Experimental studies have shown that degree of fill in the channel is known to have a significant impact on the mixing in the extruder (Bigio and Wang 1996). However, previous numerical simulations have only focused on the fully filled extruder, and therefore 
one of the contributions of this paper is to study the mixing and free-surface within simulations of a partially filled extruder.

For the purposes of the paper, a Newtonian rheological model is used. However, the SPH method can also be used to model other fluid rheology. Many non-Newtonian fluids can be modelled with a Newtonian stress tensor and a variable viscosity. As the normal SPH formulation uses a variable viscosity for each particle, this is simple to implement (Shamekhi et al. 2008; Shao and Lo 2003). It is also possible to derive an SPH formulation using a generalised Newtonian stress tensor, as shown in (Laigle et al. 2007, Rodriguez-Paz and Bonet 2004, Hosseini et al. 2007). The Lagrangian nature of SPH is an advantage when modelling visco-elastic fluids (Ellero and Tanner 2005), where the relationship between stress and strain depends on the time history of each particle.

In this paper we explore the transport and mixing properties of a co-rotating twin screw extruder with both screw elements and kneading blocks using SPH. The effect of parametric variations in geometry of both mixing elements on the nature of the fluid flow and the mixing are examined. These include varying the fill ratio, screw pitch, kneading block stagger angle and gap size between the rotating elements. Flow and mixing behaviour is also investigated for partially filled cases which are particularly well suited to the use of the SPH method because of the complex and often fragmented free surfaces that occur. The effect of the orientation handedness of the screw and kneading blocks is also investigated.

\section{Methods}

\subsection{Smoothed Particle Hydrodynamics}

The SPH formulation used for this paper is identical to the one described in Robinson et al. 
(2008) and described in Cleary (1998) and Cleary et al (2007). It is a quasi-incompressible method that uses an equation of state given by Cole (1948) and evolves the particle densities using the SPH version of the continuity equation. The traditional cubic spline kernel is used to interpolate the fluid equations across the particles and the viscous term used is described by Monaghan (2005) (Eq. 8.11). The no-slip boundaries are modelled using a single layer of boundary particles and the sharp corners are modelled using a multiple-normal technique proposed by Monaghan (2003). The geometries used in this paper were all constructed using the CAD software SOLIDWORKS and then converted to boundary particles using in-house software. The spacing between boundary particles is set to be equal to the fluid particle spacing.

The basis of the SPH method is that any continuous function $A$ can be expressed in an SPH form so that the interpolated value of this function at any position $\mathbf{r}$ becomes:

$$
A(\mathbf{r})=\sum_{b} m_{b} \frac{A_{b}}{\rho_{b}} W\left(\mathbf{r}-\mathbf{r}_{b}, h\right)
$$

where $m_{b}$ is the mass, $\rho_{b}$ is the density and $\mathbf{r}_{b}$ is the position of particle $b . W$ is a cubicspline interpolation kernel with smoothing length $h$ that approximates a Gaussian function. This smoothing kernel allows physical properties of the fluid to be converted from discrete particle data to smooth continuous interpolation fields. The gradient of the SPH function is simply given by differentiating equation (1):

$$
\nabla A(\mathbf{r})=\sum_{b} m_{b} \frac{A_{b}}{\rho_{b}} \nabla W\left(\mathbf{r}-\mathbf{r}_{b}, h\right) .
$$

For modelling of fluids with free surfaces, the continuity equation can be formulated as: 


$$
\frac{d \rho_{a}}{d t}=\sum_{b} m_{b} \mathbf{v}_{a b} \cdot \nabla_{a} W_{a b},
$$

where $\rho_{a}$ is the density of particle $a$ with velocity $\mathbf{v}_{a}$. We denote the position vector from particle $b$ to particle $a$ by $\mathbf{r}_{a b}=\mathbf{r}_{a}-\mathbf{r}_{b}$ and the velocity difference by $\mathbf{v}_{a b}=\mathbf{v}_{a}-\mathbf{v}_{b}$. This form of the continuity equation is not affected by density discontinuities such as at free surfaces and has excellent numerical conservation properties.

The SPH method used here for fluids is a quasi-compressible formulation with an equation of state specifying the relationship between particle density and fluid pressure. A form suitable for weakly compressible fluids is:

$$
P=\frac{c^{2} \rho_{0}}{\gamma}\left\lceil\left(\frac{\rho}{\rho_{0}}\right)^{\gamma}-1\right\rceil,
$$

where $P_{0}$ is the magnitude of the pressure given by

$$
\frac{\gamma P_{0}}{\rho_{0}}=100 V^{2}=c^{2}
$$

and $V$ is the characteristic or maximum fluid velocity and $c$ is the speed of sound. This means that the sound speed is ten times the characteristic speed and ensures that the density variation is less than $1 \%$ and that the flow is close to incompressible. $\rho_{0}$ is the reference density and $\gamma=7$.

The SPH form of the momentum equation becomes the acceleration for each particle $a$. The version used is from Cleary (1998): 


$$
\frac{d \mathbf{v}_{a}}{d t}=-\sum_{b} m_{b}\left\lceil\left(\frac{P_{b}}{\rho_{b}^{2}}+\frac{P_{a}}{\rho_{a}^{2}}\right)-\frac{\xi}{\rho_{a} \rho_{b}} \frac{4 \mu_{a} \mu_{b}}{\left(\mu_{a}+\mu_{b}\right)} \frac{\mathbf{v}_{a b} \cdot \mathbf{r}_{a b} \mathbf{r}_{a b}+\eta^{2}}{\rfloor}\right\rfloor+\mathbf{g}
$$

where $P_{a}$ is the fluid pressure for particle $a$ derived from the equation of state (equation 4) and $\mu_{a}$ is the particle viscosity. $\mathbf{g}$ is the gravitational acceleration vector, $\xi$ is a factor associated with the viscous term, and $\eta$ is a small parameter used to smooth out the singularity at $\mathbf{r}_{a b}=0$. With respect to the standard form of the Navier-Stokes equation, the first two terms here represent the pressure gradient term and the third term represents the Newtonian viscous stress term. The SPH viscous stress formulation used here allows for accurate momentum transfer when there are very large spatial variations or discontinuities of viscosity within the domain.

The method uses an explicit integration scheme and the simulation timestep $\Delta t$ is governed by the Courant condition:

$$
\Delta t=\min { }_{a}\left(\frac{0.5 h}{c+2 \xi \mu_{a} / h \rho_{a}}\right) .
$$

This formulation of the SPH equations applies for both single and multi-fluid applications where the different fluids are miscible. In particular, equation (6) has been formulated to allow large variations in the material viscosity on a particle by particle basis. If multiple immiscible materials are used then interface boundary conditions such as surface tension need to be considered.

Robinson et al. (2008) made a detailed comparison of the predictions of the SPH method to other numerical methods and to experiment for a two dimensional Twin Cam mixer. The SPH method was found to be more accurate in predicting the advection and mixing patterns 
in this mixer (which is equivalent to a single cross section of the three dimensional geometry used in this study).

The accuracy of this classical formulation of the SPH method has been tested (in three dimensions) by comparing to analytic solutions for Poiseuille, Taylor-Couette, combined tangential Couette and axial Poiseuille flow through an annular channel (Wittek et al. 2018), Convergence to the analytic solution was also investigated with increasing SPH resolution. Finally, Wittek et al. (2018) made comparisons between the SPH solution and an FEM solution that used a specialised treatment to handle the relative motion of the meshes due to the differential impeller motion for a pair of kneading blocks similar to the kneading block component of the impeller used in the existing study, This showed that the SPH and FEM solutions were extremely similar. Convergence, order of accuracy and issues relating to consistency arising from incomplete kernel normalization at free surfaces and boundaries are as discussed in Cleary and Monaghan (1999). Note that at the relatively low Reynolds numbers of the flows considered here that the usual 'tensile instabilities' are naturally suppressed.

The accuracy of the free surface prediction of this classical variant of the SPH method has been tested by comparison to experimental measurement for wave impact on a structure (Cummins et al. 2012), and for high speed fluid flow during cavity filling (Ha et al. 2000; Cleary et al. 2006; Cleary et al. 2014).

\subsection{Quantitative Mixing}

One of the first questions to consider in any mixing study is how to illustrate or to measure the mixing. The Residence Time Distribution (RTD) is often used to provide information on the mixing and transport properties within a Twin Screw Extruder (e.g. Kao and Allison 
1984). The RTD has the advantage that it can be measured from experiments as well as simulations; however, it does not directly measure the mixing in the device.

Another method to illustrate the mixing or transport is to track a dyed region over time. The primary advantage of this technique is that it is intuitive, directly communicates the movement of fluid and can also be used to compare experimental and simulation studies. This was the approach used to initially validate the 2D SPH predictions for the twin Cam mixer. It was also used by Bertrand et al. (2003) and Lawal and Kalyon (1995) to validate their FEM predictions. Its disadvantage is its qualitative nature, which prevents it from being used in quantitative comparisons between different mixer parameters.

A more quantitative approach, previously introduced in Robinson et al. (2008) and used by Robinson and Cleary $(2011,2012)$, uses a measure $M(\mathbf{x})$ that predicts the degree of mixing between two or more arbitrary regions that are tracked over time. This is a direct mixing measure, unlike the RTD, and can be used to quantitatively compare different mixer parameters. This approach is again used in this paper. For a given set of particles each having one of a possible $U$ colours (we use $U=2$ for this paper), the mixing measure $M(\mathbf{x})$ measures how "mixed" the colours are using a particular length scale $L . \quad M(\mathbf{x})$ is calculated at each point $\mathbf{x}$ in the domain, and the length scale defines a circular (or spherical in 3D) sub-region around each point with a radius equal to $L$. All the particles within this subregion contribute to the amount of mixing at point $\mathbf{x}$. The measure is defined as

$$
M(\mathbf{x})=\frac{\mathbf{s}_{L}(\mathbf{x}) \cdot \mathbf{s}_{g}-M_{\min }}{\mathbf{s}_{g} \cdot \mathbf{s}_{g}-M_{\min }}
$$

where $\mathrm{s}_{L}(\mathrm{x})$ is a vector of length $U$ containing the ratios of different coloured particles in the local subregion. The global ratio $\mathrm{s}_{g}$ is simply $\mathrm{s}_{L}(\mathrm{x})$ evaluated over the entire flow region 
( $\mathrm{s}_{g}$ is therefore independent of $\mathrm{x}$ ) and $M_{\mathrm{min}}$ is the minimum possible value of $\mathrm{s}_{L}(\mathrm{x}) \cdot \mathrm{s}_{g}$. The mixing measure $M(\mathrm{x})$ thus defined is a measure of how similar the colour ratios within the local sub-region are to the colour ratios present in the entire domain, and is scaled to lie in the range $0 \leq M(\mathrm{x}) \leq 1$, where 0 indicates "not mixed" and 1 is "completely mixed". The number of particles within each local sub-region must be large enough so that $\mathrm{s}_{L}(\mathrm{x})$ varies continuously with $\mathrm{x}$, and for the SPH method this is satisfied when the length scale $\mathrm{L}$ is equal to or larger than the smoothing kernel radius $2 h$.

\subsection{Finite-Time Lyapunov Exponents}

The Finite-Time Lyapunov Exponents (FTLE) (Haller 2001, Shadden et al. 2005) can also be used to quantitatively measure mixing, with the added advantage that it provides a spatial distribution of the mixing strength.

The FTLE field is a measure of the exponential stretching of the fluid and is thus directly related to the local mixing strength. Consider a line segment at time $t$ with length vector $\delta \mathbf{x}$ that moves with the flow velocity. Assume that this segment is aligned along the direction of maximum stretching of the flow. At time $t+T$ this length has moved to a new orientation and length $\delta \mathbf{X}$. The FTLE measures the exponential stretching of $\delta \mathbf{x}$ over time T.

$$
\sigma=\lim _{\|d x\| \rightarrow 0} \frac{1}{T} \ln \frac{\|\boldsymbol{\delta} \mathbf{x}\|}{\|\boldsymbol{\delta} \mathbf{x}\|}
$$

A method for calculating FTLEs from SPH simulation data was introduced for use with SPH in Robinson et al (2008) and extended to 3D and used to analyze mixing performance in a range of helical fluid mixers (Robinson and Cleary, 2012). This method is summarized below. 
To first order, the maximum separation of the two particles will occur when they are aligned along the eigenvector associated with the largest eigenvalue of $\partial \mathbf{v} / \partial \mathbf{x}$, where $\mathbf{v}$ is the flow velocity. Thus, if $\lambda_{\max }$ is the absolute value of the maximum eigenvalue of $\partial \mathbf{v} / \partial \mathbf{x}$, then

$$
\max \|\delta \mathbf{X}\|=\lambda_{\max }\|\overline{\delta \mathbf{x}}\| T
$$

where $\lambda_{\max }$ is related to the FTLE $\sigma$ by

$$
\sigma=\frac{1}{T} \ln \left(\lambda_{\max } T\right)
$$

The gradient $\partial \mathbf{v} / \partial \mathbf{x}$ can be found at a particular SPH particle $a$ by using a linear leastsquares method on the irregularly spaced particles surrounding the base particle. A linear velocity gradient is assumed around particle $a$, given by (using tensor component notation)

$$
\begin{aligned}
& v^{i}\left(x^{j}\right)=A^{i j}\left(x^{j}-x_{a}^{j}\right)+v_{a}^{i} \\
& A^{i j}=\frac{\partial v_{i}}{\partial x_{j}}
\end{aligned}
$$

If one considers a neighboring particle $b$ with an initial separation from $a$ of $\delta \mathbf{x}_{\mathrm{ab}}$, then after time $T$ the SPH simulation will have calculated the final separation $\delta \mathbf{X}_{\mathrm{ab}}$ of these particles. Using the linear velocity gradient, the final separation $\delta \mathbf{X}_{\mathrm{ab}}$, can be related to the initial separation $\delta \mathbf{x}_{\mathrm{ab}}$ by

$$
\delta X_{a b}^{i}=A^{i j} \delta x_{a b}^{j} T .
$$

In principle, only two such particles would be needed to solve Equation (13) for the 
velocity gradient components $A^{i j}$. However, since the velocity gradient is very sensitive to errors in the particle separations, we use all of the particles within the smoothing length (2h) of the base particle $a$, assuming that the velocity gradient will be constant over this volume. Using these $\mathrm{N}$ particles, $A^{i j}$ is found by minimising the sum

$$
\sum_{b}^{N}\left(\delta X_{b}^{i}-A^{i j} \delta x_{b}^{j} T\right)^{2} .
$$

Once the velocity gradient is found, its maximum eigenvalue can be calculated and substituted into equation (11) to give the FTLE of particle $a$. This entire process is then repeated for all the SPH particles within the domain so as to give the spatial variation of the FTLE.

\section{Extruder Configuration and Simulation Conditions}

The geometry of the twin screw extruder is based on the $2 \mathrm{D}$ cross-section depicted in Figure 1a. The outer casing of the mixer is a double barrelled enclosure, containing two mixing impellers. The centres of each barrel (and each screw element) are separated by $\mathrm{C}=$ $40.25 \mathrm{~mm}$, and the radius of each barrel is $\mathrm{R}_{\mathrm{b}}=26.5 \mathrm{~mm}$.

The impeller cross-section is defined by $R_{t}=25 \mathrm{~mm}$ and $R_{s}=13.75 \mathrm{~mm}$ which are the maximum and minimum radii of the impeller cross-section. The tip angle is $\alpha=11.61$ degrees. The radius of each tip and the separations of the elements from those of the other impeller are chosen so that the minimum gap between them is $g=1.5 \mathrm{~mm}$. The distance between the impeller tips and the outer casing is $1.5 \mathrm{~mm}$. The impeller is made up of one or more elements that have this cross-sectional shape (as shown in Figure 1a). 
This cross-section can be used to generate two commonly used impeller elements:

1. A fully-flighted screw, seen in Figure $1 \mathrm{~b}$ in a side view, which is swept out by continuously rotating each screw cross-section while moving along the rotation axis. The relevant parameters for this element are the screw pitch length $p=50 \mathrm{~mm}$ and the extruder length $\mathrm{L}_{\mathrm{ff}}$.

2. A set of kneading blocks, which are generated by rotating each cross-section in discrete jumps to create a set of discs for each kneading block. The parameters for the kneading blocks are the individual disc width $d=12.5 \mathrm{~mm}$, the total length of the kneading block element $\mathrm{L}_{\mathrm{ke}}$, and the disc stagger angle $\beta=30$ degrees, which is the difference in rotation angle between subsequent discs (see Figure 1c).

This paper examines the mixing in a twin screw extruder due to these elements. The three base extruder geometries used are:

1. A combination extruder, with each barrel comprising a central kneading blocks section of length $\mathrm{L}_{\mathrm{ke}}=137.53 \mathrm{~mm}$ surrounded by two screw elements, both with length $\mathrm{L}_{\mathrm{ff}}=66.67 \mathrm{~mm}$. Figure 2 shows this geometry.

2. Two screw elements, each of length $\mathrm{L}_{\mathrm{ff}}=75 \mathrm{~mm}$. Figure 8 shows this geometry.

3. Two kneading blocks of length $\mathrm{L}_{\mathrm{ke}}=75 \mathrm{~mm}$. Figure 7 shows this geometry.

In all cases the axis of rotation is along the local $z$-coordinate axis. Periodic boundary conditions are used at either end of the extruder in this direction. Particles at each end are able to see a periodic copy of the particles at the other end which ensures continuity of all flow properties across the periodic boundary. Particles passing through the periodic boundary are translated to the other end of computational domain. The use of such a 
periodic boundary condition is equivalent to having an infinitely long mixer with sequential copies of the base impeller unit repeated along the periodic (axial) direction. The mixer configuration is either partially or completely filled with a viscous (Newtonian) fluid. The rotation speed of each impeller is $\omega=1 \mathrm{rad} / \mathrm{s}$.

The $\mathrm{SPH}$ resolution is spatially uniform with a particle spacing of $\delta \mathrm{p}=0.75 \mathrm{~mm}$ (half the gap distance of $\mathrm{g}=1.5 \mathrm{~mm}$ ), and a smoothing length of $\mathrm{h}=1.5 \delta \mathrm{p}$. The smoothing length defines a radius of interaction around each SPH particle which is $2 \mathrm{~h}$. This is also the length scale $(\mathrm{L}=2 \mathrm{~h})$ used for the mixing measure calculations. So the resolution of the simulation, $\mathrm{h}$, is also the length scale used to calculate the quantitative mixing measures.

The fluid properties matched those of water with a reference density of $\rho_{0}=1000 \mathrm{~kg} / \mathrm{m}^{3}$ and the dynamic viscosity of $\mu=8.9 \times 10^{-4} \mathrm{~Pa} \cdot \mathrm{s}$. The speed of sound in the SPH equation of state was set to 10 times the maximum velocity of the fluid. In the fully filled cases this is given by the peripheral speed of the impeller $\left(\mathrm{v}_{\max }=\omega \mathrm{R}_{\mathrm{t}}\right.$ which in this case is 0.025 $\mathrm{m} / \mathrm{s}$ ). In the partially filled cases, the controlling speed is from the speed of waves arising from gravity which are controlled by the depth of fluid between shell and narrowest part of the impeller, which is $R_{b}-R_{s}=12.75 \mathrm{~mm}$, and gives a characteristic wave speed of $\mathrm{v}_{\max }=$ $0.5 \mathrm{~m} / \mathrm{s}$. Using these maximum speeds to set the speed of sound ensures that density variations were less than $1 \%$ so as to satisfy the quasi-incompressible constraint. Using $g=$ $1.5 \mathrm{~mm}$ as the characteristic length (i.e. $\mathrm{L}_{\mathrm{re}}=\mathrm{g}=1.5 \mathrm{~mm}$ ) and the maximum velocity of the mixer geometry as the characteristic velocity $\mathrm{v}_{\max }$, the Reynolds number of the flow is $\mathrm{Re}=$ $\mathrm{L}_{\mathrm{re}} \omega \mathrm{R}_{\mathrm{t}} \rho / \mu=42$ which indicates that it is laminar in nature and viscous dominated.

In many extrusion applications the fluid viscosity can be much higher than the value used 
here, however, the flow behaviour becomes independent of viscosity when it is larger (Robinson and Cleary, 2008). There are two or three competing timescales depending on whether the mixer is fully or partially filled which control the dynamics. These arise from viscous forces, from deformation driven by the motion of the impellers and from deformation arising from flow in response to gravity. The characteristic speed associated with impeller motion is $0.025 \mathrm{~m} / \mathrm{s}$ and for the gravitational driven waves in the partially filled extruder cases is $0.5 \mathrm{~m} / \mathrm{s}$. For very viscous fluids, the viscous timescale is sufficiently fast that the flow structure equilibrates to each incremental change in impeller orientation or deformation driven by gravity, meaning that the flow is only controlled by the rate of geometric change or by gravity in the partially filled cases and is therefore independent of viscosity. The viscosities required to achieve this independence are $3.75 \times 10^{-4} \mathrm{~Pa} \cdot \mathrm{s}$ for the fully filled configuration and $6.38 \times 10^{-2} \mathrm{~Pa} \cdot \mathrm{s}$ for the partially filled cases. The viscosity used here is sufficient to be representative of more viscous fluids for the fully filled cases. For the partially filled cases there will be some viscosity dependence in the specific results reported here since the viscosity is above the viscosity independence limit.

The fluid particles were initially placed within the mixer on a regular grid pattern. The initial particle density is set slightly higher than the reference density $\rho_{0}$ so as to produce an initial over-pressure and ensure that for the completely filled simulations the fluid did indeed fill the space between the extruder barrel and the impeller.

\section{Mixing in an Extruder with Screw Elements and Kneading}

\section{Blocks}

This section presents the results from an SPH simulation of the full twin screw mixer 
geometry with the combined screw elements and kneading blocks. In order to analyse the transport and mixing within the device, two Lagrangian volumes of fluid are chosen:

- Volume One is defined as all the fluid to the left of the $x=0$ plane (vertical plane between the two screw elements) at $\mathrm{t}=0 \mathrm{sec}$. This volume is all the fluid in the left hand barrel of the mixer, and as the simulation proceeds, this volume of fluid will be transported across the centre plane of the mixer. The mixing of this volume is therefore representative of the cross-channel mixing.

- Volume Two is all the fluid within the two planes $z=33 \mathrm{~mm}$ and $z=33+25 \mathrm{~mm}$, where $z=33 \mathrm{~mm}$ is a plane in the middle of the first screw module. This volume will be mixed with the remainder of the fluid in the positive and/or negative axial direction, and is therefore representative of the overall axial mixing in the device.

Two different set of volumes are used in this analysis in order to show the mixing behaviour in the two key directions (axial and cross-chamber) which are quite different in their nature. It is important that the anisotropy of the mixing be able to be evaluated. The use of the different volumes and directions of mixing analysis enables this.

Figure 3 shows the evolution of Volume One over $0<\mathrm{t}<4 \mathrm{sec}$, which corresponds to approximately $3 / 4$ of a single revolution of the impellors. The surface of the Lagrangian volume is initially shaded with an arbitrary red and blue checkerboard pattern in order to see the fluid transport within the volume. The cross-channel mixing shown by the transport of Volume One is substantially different around the screw elements than the region surrounding the kneading blocks. The screw elements provide minimal mixing and mainly serve as a bulk transport process in the positive $z$ direction (down the length of the mixer). There is a small amount of cross-channel mixing, and this can be seen at the bottom of each 
plot as the initially vertical interface between the fluid in the two barrels is stretched out in the direction of fluid flow around the two screws. However, the mixing in the screw elements is minor compared to that surrounding the kneading blocks. Over the course of the 3/4 revolution shown in Figure 3, a large proportion of the volume surrounding the kneading blocks is transported into the opposite barrel, and the checkerboard pattern is quickly disrupted due to the mixing processes within the volume. There is also some evidence of axial mixing due to the kneading blocks, as shown by the increasing axial spread of fluid in the right hand barrel over time. However, this is more clearly shown by the transport of Volume Two.

Figure 4 shows the evolution of Volume Two over the same time period $(0<t<4 s)$. The transport of this volume of fluid shows the axial mixing within the twin screw mixer. Once again the surface of the volume is shown and coloured with an arbitrary pattern in order to visualize the mixing. In this case the fluid in each barrel is given a different colour in order to show some of cross channel mixing and to relate this to the transport of Volume One. As seen previously with Volume One, the mixing of Volume Two around the screw elements is minimal and this section only serves to transport the fluid to the main mixing region (i.e. the kneading blocks). There is little deformation of the surface of Volume Two in the axial direction. Once the fluid reaches the kneading blocks (see the snapshot at $t=2 \mathrm{~s}$ ) it quickly starts to deform and stretch. The fluid in the right hand barrel is pulled forward in the positive $z$ direction while the fluid in the left hand barrel is held back. At the same time, there is a fast rotation of fluid clockwise around the mixer, so that the red fluid is transported up and across to the right hand barrel, which the blue is moved down and across to the left hand side (this is obscured in the figure due to the camera angle). At $t=4 \mathrm{~s}$ some reasonable stretching of Volume Two has occurred, but the amount of mixing is still 
relatively small.

Figure 5 shows evolution of the average mixing $\langle\mathrm{M}\rangle$ over time for both fluid volumes using the mixing measure $\mathrm{M}$ introduced previously. Since periodic boundary conditions are used in the axial direction, all the fluid has an identical residence time in the mixer and therefore $\langle\mathrm{M}\rangle$ gives the amount of mixing for a characteristic volume of fluid within the mixer. The change of $\langle\mathrm{M}\rangle$ with time therefore provides a measure of the overall rate of mixing. The two "colours" or sets of particles (i.e. $U=2$ ), used to evaluate the mixing measure were (1) those belonging to either Volume One or Two, and (2) those that did not belong. That is, the visible and invisible particles shown in Figure 3 and 4 . This measure is zero for fully unmixed and unity for fully mixed. This allows for a quantitative comparison of the cross-channel and the axial mixing rates. In some cases a small non-zero initial value of the measure is observed due to aliasing at the interface between materials. This occurs because there are always some of the averaging cells that are part filled with both materials even in a perfectly unmixed system. The precise value of this initial value depends on the degree of alignment of the averaging cells with the location of the interface.

In practice this is small and does not affect the quantification of the mixing. At small times $(\mathrm{t}<5 \mathrm{~s})$, the average mixing rate of Volume Two is much lower than Volume One. During much of this time, Volume Two is still in the screw section of the mixer and has not yet reached the kneading blocks. Meanwhile, approximately half of Volume One has moved into the kneading block section, and this strongly increases the average mixing for this fluid volume. While the mixing rate of Volume Two does increase once the fluid reaches the kneading blocks, it is always lower than that of Volume One and it is clear from Figure 5 that the mixing in the cross-channel direction is stronger than along the axis of rotation. 
The mixing shown in Figures 3-5 is controlled by the fluid velocity distribution within the mixer. Figure 6 shows the structure of the flow in vertical cross-sections through the screw and kneading block sections of the mixer. Figure $6 \mathrm{~d}$ shows the location of two planes, one located within the screw elements and the other within the kneading blocks. The other three panels of this figure show the spatial distribution of flow properties these two planes. Figure 6a shows the distributions of the FTLE field which quantifies the spatial contributions to the mixing, Figure $6 \mathrm{~b}$ shows the axial (z-axis) velocity, and Figure $6 \mathrm{c}$ shows the cross-channel velocity in the $x-y$ plane. In each panel the top plot shows the plane in the screw elements, while the bottom plot shows the plane in the kneading blocks.

The FTLE plots show a significant difference between the two sections of the mixer. In the screw element the FTLE values are very low (less than 0.2 ), indicating that the stretching of fluid elements in this section is occurring relatively slowly. Within the kneading blocks the average FTLE is much higher. This plot (Figure 6a) shows a complex pattern of FTLE over the twin screw domain, with high values reaching up to 0.7. The variable FTLE field represents the stretching and folding of fluid elements that is generated by differential fluid flow produced by the relative rotation of the kneading blocks. In general, the high values of FTLE seen in this section are indicative of high mixing rates, confirming the qualitative results seen in the visualizations of Volume One and Volume Two.

The fluid velocity in the axial direction is shown in Figure 6b. For the screw section, this velocity field is almost homogenous, showing that the screw acts as a bulk fluid transport element. Within the kneading blocks, the axial velocity is highly variable. In the crosssection shown here, there is a significant backflow of fluid down the bottom of the mixer. This backflow is quite rapid and has a much higher speeds than the average transport 
velocity of the fluid along the length of the mixer.

The two cross-sections in Figure 6c show the velocity of the fluid within the $x-y$ plane. In both the screw and kneading block sections the fluid moves in a clockwise direction around the outside of the mixer. However, the average $x-y$ velocity magnitude within the kneading blocks is significantly higher than in the transport elements, reaching a maximum of 36 $\mathrm{mm} / \mathrm{s}$ just behind the tip of each block. This is approximately 3 times higher than the peak $x-y$ velocity in the screw elements.

\section{Independent Mixing Behaviour of Screw Elements and Kneading Blocks}

The previous section examined the mixing within a twin screw mixer containing a combination of screw and kneading block impellor sections. This section investigates in more detail the mixing surrounding each impellor type in isolation, allowing us to identify the independent effect of each impellor type on the fluid mixing.

Once again, two different volumes, Volume One and Volume Two, are used to visualize the cross-channel and axial mixing. The extents of both volumes are identical to that described in the previous section. Figure 7 shows the evolution of Volume One and Volume Two in a twin screw mixer containing only kneading block impellors. The top row shows the transport of Volume One, which is representative of the cross-channel mixing in the mixer. Due to the direction of rotation of the impellors this volume of fluid is moved around the mixer in a clockwise fashion, with the fluid in the top half of the left barrel moving into the top half of the right barrel. The checkerboard pattern is deformed rapidly over time, showing a significant amount of mixing occurring internally. The bottom row of Figure 7 
shows the transport of Volume Two, which is representative of the axial mixing. After the first frame $(t=3.2 \mathrm{sec})$ the blue fluid that is originally in the left hand barrel has been transported clockwise around the impellors so that it is now located across the top of both barrels. At the same time, the blue fluid has also been stretched out diagonally, so that the fluid left in the left barrel has moved further down the axis of the barrel, while the fluid in the right hand barrel has remained at approximately the same location on the $z$ axis. A similar process has occurred to the red fluid that was originally in the right hand barrel, except that it has moved further down the length of the mixer.

In general, if one considers a representative area of fluid that is orientated with the $x-y$ plane, the primary effect of the mixing flow surrounding the kneading blocks is to stretch out and reorient this area diagonally across the mixer, along the same line that is formed by connecting the tips of each kneading block. However, it should be noted that this is a general trend rather than a smooth, continuous process due to the discrete nature of the blocks. Since the angle changes of the kneading blocks are discontinuous, the flow structures that this creates cause an increasing amount of fragmentation of the fluid volume. This is seen most clearly in the third frame $(t=6.4 \mathrm{sec})$, where the volume has now been spread out and fragmented over the entire domain.

Figure 8 shows the evolution of Volume One and Volume Two in a twin screw mixer containing only screw elements. The top row shows three snapshots from the transport of Volume One. The clockwise movement of the fluid around the impellors causes the initially vertical interface between the fluid in the left and right hand barrels to be elongated and rotated to lie on a diagonal. Besides a minor amount of mixing that occurs near the centre line, this is the only mixing process that operates in the cross-channel direction. The bottom 
row of Figure 8 shows the evolution of Volume Two. In general, Volume Two is transported as a bulk down the main axis of the mixer, and in the first two frames it is impossible to distinguish any variation in the surface of the volume. However, the third frame is taken from a top down angle, and from here it can be seen that the surface near the centre line of the mixer is being deformed and elongated away from the rest of the bulk volume. This is due to slightly higher/lower axial velocities directly above/below the centre line of the mixer, and only becomes obvious after a whole rotation of the impellors.

The primary difference between the flow near the screw elements and that surrounding the kneading blocks is that the transport and mixing of both fluid volumes is significantly slower and occurs with little or no fragmentation of the volume. This is due to the smooth, continuous nature of the screw impellors, which set up a homogenous flow field that generates little or no mixing (with little stretching or folding motion). The primary consequence of the screw elements is therefore to transport the fluid along the mixer, rather than to perform any substantive mixing.

In order to perform a quantitative comparison between the cross-channel and axial mixing within each mixing module, Figure 9 shows the average mixing versus time for both impellor geometries and both fluid volumes, using the average mixing $\langle M\rangle$ that was introduced earlier in this paper. Figure 9a shows the results for the kneading blocks. Of the two fluid volumes considered, the mixing rate of Volume Two is clearly the quickest, saturating at an average mixing value of approximately 0.9 after $30 \mathrm{sec}(\sim 5$ impellor rotations). This mixing of Volume One does not saturate during the time period shown, although by the end of the simulation $(t=60 \mathrm{sec})$ the value of $\langle M\rangle$ is close to 0.9 . This plot shows that the dominant mixing mechanism in the kneading blocks occurs in the axial, 
rather than the cross-channel direction.

Figure $9 \mathrm{~b}$ shows $\langle M\rangle$ versus time for the screw elements. Consistent to what has already been seen in the fluid volume visualisations, the mixing rate of the screw elements is far slower than for the kneading blocks, with the maximum mixing measure for both volumes not having yet reached 0.11 by the end of the simulations. The initial value of $\langle M\rangle$ is determined by the interfacial area between the fluid volume under consideration and the rest of the fluid in the mixer. In this case, the interfacial area is much larger for Volume Two, so this volume starts with a higher initial value for $\langle M\rangle$. Over the first revolution of the impellors $(0<t<6.28 \mathrm{sec})$, the rate of mixing for Volume One is significantly higher and quickly catches up with that of Volume Two. For $t>10 \mathrm{sec}$, the increase of $\langle M\rangle$ becomes linear for both representative volumes, with the (constant) rate of mixing for Volume Two being slightly higher than for Volume One. So the axial mixing rate for the screw elements is slightly higher than that in the cross-channel direction. This means that the mixing for this impeller type is nearly isotropic. This contrasts with the mixing for the kneading blocks which is not at all isotropic (being axially dominated) and which occurs orders of magnitude faster.

\section{Influence of Impeller Design Parameters on Mixing}

Now that the comparative mixing between the screw elements and kneading blocks have been established, this section explores the variation of the transport and mixing with changes in the geometric parameters that define the impellor shapes. As in the previous section, the separate screw element and kneading block geometries are also used for these comparisons. The average mixing measure $\langle M\rangle$ is used to quantify mixing and is the basis for comparison. 
Figure 10 shows the effects of varying the pitch length $p$ of the screw elements on the mixing. Although the role of the screw elements is predominantly that of transport, they do still contribute to mixing. A longer pitch $p$ means that the length of one rotation of the screw is increased, resulting in an increased distance between subsequent flights (tips) of the screw. The bulk movement of fluid along the mixer is unaffected, as the rotation speed of the screw element is constant. However, the mixing results for both Volume One and Two are consistent, and show that an increase in the $p$ leads to an increase in mixing rate. The effect is slightly stronger for the axial mixing. The axial mixing is due to changes in the axial velocity near the centre-line of the mixer (where the twin screws intermesh). An increased sensitivity of the axial mixing due to a change in $p$ is consistent with this, as a longer $p$ changes the angle at which the two screws intermesh which leads to an intensification of the flow field in this region.

Figure 11 shows the effect of the stagger angle $\beta$ between subsequent kneading discs on the progress of overall mixing state. The range of angles considered here range from 30 degree (the $\beta$ used in the original geometry) up to the most extreme case of 90 degree, where each kneading disc is perpendicular to the previous. In general, the original $\beta=30$ degree leads to the best mixing results. An increase beyond this value leads to a decrease in the rate of both axial and cross-channel mixing. However, this trend breaks down at $\beta=90$ degree. For this case, the kneading discs are no longer structured as a "discrete screw" shape but then are simply alternating between two orthogonal block orientations. This case behaves markedly differently with significantly reduced axial mixing and much more rapid azimuthal or cross-chamber mixing. This is a consequence of the force exerted on the fluid by the kneading blocks predominantly now acting in the plane perpendicular to the rotational axis of the mixer. These results are consistent with those by Lawal and Kalyon (1995), who 
visualised the transport of a "blob" of fluid around the kneading block geometry with $\beta=30$ and 90 degree, and found that the blob experienced minimum spreading in the axial direction for the 90 degree case. For the lesser block angles, the changes are much more moderate with both the cross-chamber and axial mixing rate decreasing slightly with increasing block stagger angle $\beta$. In terms of having a good balance between the directions of mixing and having the fastest mixing rates, the base case $\beta=30^{\circ}$ provides the best performance of the cases considered.

Figure 12 (screw elements) and Figure 13 (kneading blocks) show the effect of varying the gap size $g$ between the two rotating impellors. The general effect of increasing $g$ is consistent across both geometries and both directions considered. An increase in the $g$ leads to an increase in the mixing in both the cross-channel and axial direction with the magnitude of the increase larger for the screw elements and very weak in the axial direction for the kneading blocks. The effect is generally stronger in the cross-channel direction. The results for the kneading elements (as shown by visualisation of Volume 1 in Figure 7) are consistent with the earlier findings by Rios et al (1998), using a 2D Boundary Element Method, that the flux of fluid passing between the two barrels of the mixer is greater with an increase in the $g$.

\section{Influence of Partial Fill on Flow and Mixing Performance}

\subsection{Effect of partial fill for each impeller component}

One key advantage of the SPH method is the ability to easily model free-surface flow. Since twin screw extruders are normally run in starvation mode, a free-surface is often a flow feature which needs to be considered. This section describes the results of SPH simulations of mixing in a twin screw extruder with a varying level of fluid fill. The same 
impellor geometries used earlier are considered.

The primary role of the screw elements is to convey fluid along the length of the mixer. It is therefore most often used as a first module of a twin screw extruder, in order to convey the fluid to the primary mixing elements, which in this case are the kneading blocks. This means that the fluid levels in the screw elements can vary from almost empty to $100 \%$. We therefore consider a wide range of fill levels in this paper for the screw component models. Conversely, the kneading blocks are usually operated using a high fill level in practical configurations, so only levels greater than $80 \%$ are considered here.

Figure 14 show snapshots of the fluid distribution and free surface structure for three different fluid fill levels of $25 \%, 50 \%$ and $75 \%$ for the screw element only configuration. In each case the shape of the free-surface is shown and for clarity the screw geometry and twin screw housing are made transparent to allow the fluid surface structure to be seen. The first noticeable feature for all fill levels is that there is no fluid between the outermost edge of the screw blade (the tip) and its neighboring screw impellor. Therefore, for this particular $g$ and fluid resolution, this gap acts as an impenetrable barrier for the fluid.. For a fill level of $25 \%$, the lower section of each barrel is filled with fluid being pulled upward on the left side of each barrel by the ascending motion of each screw. The screw blade separates this fluid into separate pools and there is little ability for fluid to move from one of these pools into the next. Quite large coherent volumes of fluid are also found around the centerline of the upper part of the mixing chamber. This is above the point of closest approach of the screws whose constriction retards the fluid and prevents it from escaping downward with one screw pulling the fluid down while the other screw lifts it back up. At a fill level of $50 \%$ the heights of the free surface in the lower flooded parts of the barrel are higher and 
the volume of the upper central volumes is increased to the point where some flow over the top of the screw (see as the thin streams in the upper right of Figure 14b) is observed. By a fill level of $75 \%$ these fluid regions are all contiguous and fluid circulates freely around the screws in each of the barrels with a $25 \%$ open void space at the top of the mixer.

To explore the effect the partial filling on the mixing, Figure 15 shows the progressive mixing measure versus time for the screw element at different fill levels. The mixing rate is much faster in both cross-chamber and axial directions for all the partial fill levels compared to that for the fully filled mixer (100\%). This is a consequence of the fluid having significant extra mobility when the mixer is only partially filled enabling much faster mixing. For the cross-channel mixing (Volume One) the mixing behaviour is quite strongly dependent on the fill level. The $25 \%$ and $75 \%$ fill levels have fairly similar overall mixing behaviour; albeit with the $25 \%$ fill having much faster mixing earlier on whereas the $75 \%$ case has relatively faster mixing after about $15 \mathrm{~s}$. The slowing of the mixing rate at longer times for the $25 \%$ fill level relates to the structure of the fluid which is divided into separate free surface pools at the bottom of the barrels by the screw blade. Mixing within each of these pools is quite fast, enhanced by the presence of significant free surface area (leading to faster mixing early on), but there is limited mixing of fluid between the pools, limiting both the rate and extent of the long term mixing. The $50 \%$ fill level has the highest mixing rate over the full simulation, clearly demonstrating that a fill level of around $50 \%$ appears optimal for cross-chamber mixing.

The axial mixing (using Volume Two) is shown in Figure 15b. All the partial fill cases produce fairly similar mixing behaviour which is significantly faster (by a factor of around three) than for the fully filled case. The presence of the free surface modifies the flow 
pattern in the mixer and significantly enhances the mixing behaviour. The $25 \%$ fill level is mildly weaker than the other two cases with divergence starting to occur after $40 \mathrm{~s}$ as the restriction to global mixing between the pools separated by the screw blades starts to impact on the cumulative amount of mixing. The $75 \%$ fill level is only marginally slower than for the $50 \%$ fill level. This suggests that for axial mixing that a fill level around the range of $50-75 \%$ is likely to be optimal. The axial mixing is less sensitive to the fill level than is the cross-barrel mixing.

Figure 16 shows the fluid free-surface in the kneading block simulations for fill level of $80 \%$ and $90 \%$. For clarity the free-surface has been made transparent so that any internal free surface structure can be identified. Since the fill level is significantly higher here as compared to the screw cases results, the amount of free-surface is much smaller. The regions void of fluid occur mostly at the trailing edge of the kneading block tips as they rotate around the mixer barrels. Once again it can be seen that there is no fluid between the kneading block tips and the mixer barrel.

The quantitative mixing results for the variable fill level for the kneading block configuration are shown in Figure 17. The impact of these fill levels variations is relatively smaller than for the lower fill levels considered for the screw elements. For the crosschannel mixing, a fill level of $80 \%$ leads to around a $25 \%$ increase in the mixing rate (based on the time taken to reach a mixing level of $\langle M\rangle=0.9$ ). At a fill level of $90 \%$ the crosschamber mixing is almost the same as for the fully filled variant. So small areas of fluid free surface in this kneading configuration are not able to materially affect the mixing behaviour. For the axial mixing, the effect is even weaker with an increase in mixing rate of $16 \%$ for the $80 \%$ fill level and only a very marginal increase for a $90 \%$ fill level. So overall, 
these results show that for the fill level ranges typically used in the kneading block section of a twin screw extruder that there is little advantage to be gained from running the kneading blocks less than completely filled. However, it is clear that the presence of a freesurface is generally beneficial to the mixing process in a twin screw mixer and is therefore a factor that ought to be considered in the design process.

\subsection{Effect of relative handedness of successive impeller elements}

Finally, returning to the first configuration with both screw elements and kneading blocks, we consider the effect of the stagger direction (handedness) of the impeller components. Carneiro et al. (1999) studied twin screw extruder experiments with different combinations of screw elements and kneading blocks. They observed that the flow patterns present in the mixer (run in starvation mode; that is, not completely filled) are highly dependent on the presence of helix or stagger angles downstream that are oppositely directed to those that are upstream. This creates active resistance to forward flow in the downstream elements, slowing the axial transport and causing a build-up of fluid starting at the point of handedness reversal and more completely filled sections upstream of this point.

The steady state fluid distribution predicted by the SPH method within the mixer is shown in Figure 18 when the handedness direction is consistent along the mixer length, and is shown in Figure 19 when there is a transition in handedness at the start of the kneading blocks. In Figure 18 the kneading blocks have a right-handed stagger ( $\beta=30$ degrees) which is consistent with the right-handedness of the screw flights upstream. The predicted fluid distribution is broadly constant along the axial length of the mixer. Since the kneading blocks are the dominant contributors to the overall mixing the low fill level of fluid in this part of the mixer will lead to relatively poor overall mixing outcomes. In contrast, the 
extruder shown in Figure 19 has kneading blocks with $\beta=-30$ degrees (left handed stagger), which oppose the right handed screw. The reversal of the kneading block handedness reduces the axial transport rate within this section resulting in a build-up of fluid there. This concentrates the available fluid in the kneading block section, which then significantly improves its mixing efficiency. The mixing contribution of the screw element (already smaller as shown in Figure 9) is reduced further by the resulting very low fill level in the screw section. This means that the role of the screw element becomes almost solely one of transport. It efficiently delivers fluid to the kneading elements which then perform almost all of the mixing by stretching and folding the fluid according to the complex velocity field generated by the kneading blocks as shown in Figure 6a.

This differential axial transport behaviour is critical to twin screw extruder operation. It allows atmospheric pressure feed of fluids and/or granular materials, with the screws acting predominantly as transport elements to deliver the material to the regions of opposite handedness where densification and compaction then occurs allowing improved processing and mixing downstream of this point.

This effect of the handedness change is quantitatively shown in the mixing plots in Figure 20. The cross-chamber mixing is significantly improved (Figure 20a) for the impeller with a negative kneading $\beta$ (labelled as counter rotation in the figure legend) with a nearly $50 \%$ reduction in time to reach $\langle M\rangle=0.8$. The mixing rate in the axial direction (Figure 20b) is also improved although to a much smaller degree. Eventually the degree of mixing catches up due to the infinite residence time arising from the use of a periodic domain in the axial direction. In industrial mixers, with finite residence time, the faster mixing rates observed with the use of opposing handed impeller elements will also manifest as large differences in 
the extent of mixing of the final product.

\section{Conclusions}

SPH based numerical simulations of Twin Screw extruders have been used to gain insight into their mixing and transport properties and how these are affected by changes in impeller geometry and fluid fill level. The SPH method was used to simulate the fluid, due to its easy incorporation of free-surfaces and the avoidance of numerical difficulties relating to the complex intermeshing geometry of the impellers, as well as its general suitability for predicting mixing arising from its Lagrangian nature. Mixing was quantified using the FTLE method and using one based on the degree of homogeneity of different initial colour distributions which allows mixing to be measured independently in the cross-chamber and axial directions.

In order to better understand the mixing processes in a Twin Screw extruder, the fluid transport in and around two different impeller types comprising of screw elements or sets of kneading blocks staggered by a constant angle were investigated. The mixing around the screw elements was found to be much slower than for the kneading blocks and their main function was identified as being to transport the fluid along the length of the mixer. The kneading blocks function as the primary mixing elements, generating complex velocity fields comprised of strong components both in the cross-sectional plane of the mixer and backwards and forwards along the axis of rotation. The velocity field around the kneading blocks generates strong mixing in both directions with the axial direction being largest.

The effects on the mixing of changes in the geometric parameters that define each impeller element type were evaluated. These included the screw pitch length $p$, kneading block 
stagger angle $\beta$ and gap size $g$ between the impellors. Increasing $p$ causes the mixing in both the axial and cross-channel directions to strengthen. Increases in $\beta$ in general result in slight reductions in mixing rate, except for an angle of $90^{\circ}$ for which large increases in cross-chamber mixing were observed but at the cost of significant reductions in the axial mixing rate. The gap size $g$ was positively correlated with the cross-channel mixing, but had little or no effect on the axial mixing for the kneading blocks. The increase in mixing rate with $g$ was stronger for the screw elements.

The use of SPH to simulate the twin screw mixing also enabled investigation of the effect of fill level of the fluid in the mixer. It was found that the presence of a free-surface had a generally positive effect on mixing rates, except for very low fill levels where isolated pools of fluid formed between screw blades. This improved short term mixing within these pools but restricted global mixing between adjacent pools. For the screw elements the highest mixing rate was found for a $50 \%$ fill level. In practice, any such enhancement of the mixing rate needs to be balanced against the necessarily reduced amount of fluid being mixed. These results suggest that the amount of fluid free surface could be a useful design factor to consider in terms of improving mixing efficiency.

Finally, the effect of the consistency of the handedness of the screw and kneading elements was investigated for partially filled configurations. When impellers use the same forward direction for the screw spiral and for the stepped kneading blocks then the fluid and void distribution within the extruder was found to be uniform in the axial direction. However, when the direction of $\beta$ was opposite to that of the screw (so the elements have the opposite handedness) then the axial fluid flow was strongly retarded in the kneading section leading to a significant concentration of fluid there. This leads to a compaction of the available 
fluid in the kneading block section, which significantly improves its mixing efficiency. This means that the role of the screw elements is almost solely one of a transport. They efficiently deliver fluid to the kneading elements which then perform almost all of the mixing by stretching and folding the fluid according to the complex velocity field that they generate in the surrounding fluid.

\section{Compliance with Ethical Standards}

The authors declare that no conflict of interest exists.

This article does not contain any studies with human participants or animals performed by the author.

No individual participants were included in the study so there are no subjects for which informed consent requirements arise.

\section{Acknowledgements}

The authors would like to thank Matt Sinnott for his assistance in constructing the CAD models of the extruder. M.R. gratefully acknowledges research support from the EPSRC Cross-Disciplinary Interface Programme (EP/I017909/1).

\section{References}

Altomare, C., Crespo, A. J. C., Rogers, B. D., Dominguez, J. M., Gironella, X., \& GómezGesteira, M., Numerical modelling of armour block sea breakwater with smoothed particle hydrodynamics. Computers \& Structures, 130, 34-45, 2014. 
Avalosse, T. and Rubin, Y., Analysis of mixing in co-rotating twin screw extruders through numerical simulation. In SPE/ANTEC 1999 Proceedings, pages 322+. CRC Press, 1999.

Bertrand, F., Thibaulta, F., Delamareb, L., and Tanguya, P. A., Adaptive finite element simulations of fluid flow in twin-screw extruders. Computers \& Chemical Engineering, 27(4), 491-500, April 2003.

Bigio, D. and Wang, K., Scale-up rules for mixing in a non-intermeshing twin-screw extruder. Polymer Engineering \& Science, 36(23), 2832-2839, 1996.

Carneiro, O. S., Caldeira, G., Covas, J. A. Flow patterns in twin-screw extruders, Journal of Materials Processing Technology, 92-93, 309-315, 1999.

Cheng, H., and Manas-Zloczower. I., Distributive mixing in conveying elements of a zsk53 co-rotating twin screw extruder. Polymer Engineering \& Science, 38(6), 926-935, 1998.

Cleary, P. W., and Monaghan, J. J., Conduction modelling using Smoothed Particle Hydrodynamics, J. Comp. Phys., 148, 227-264, 1999.

Cleary, P. W., Ha, J., Prakash, M., and Nguyen, T., 3D SPH Flow Predictions and Validation for High Pressure Die Casting of Automotive Components, App. Math. Model., 30, 1406-1427, 2006.

Cleary, P. W, Prakash, M., Ha, J., Stokes, N., Scott C., Smooth Particle Hydrodynamics: Status and future potential. Progress in Computational Fluid Dynamics. 7, 70-90, 2007.

Cleary, P. W., Savage, H., Ha, J., and Prakash, M., Flow analysis and validation of numerical modelling for a thin walled High Pressure Die Casting using SPH, 
Computational Particle Methods, 1, 229-243, 2014.

Cole R. Underwater Explosions. Princeton, NJ: Princeton University Press; 1948.

Connelly, R. and Kokini, J., Examination of the mixing ability of single and twin screw mixers using $2 \mathrm{~d}$ finite element method simulation with particle tracking. Journal of Food Engineering, 79(3), 956-969, April 2007.

Cummins, S., Silvester, T.B., and Cleary, P.W. Three Dimensional Wave Impact on a Rigid Structure using Smoothed Particle Hydrodynamics, Int. Journal for Numerical Methods in Fluids, 68, 1471-1496, 2012.

de Graaf, R. A., Woldringh, D. J., and Janssen, L. P. B. M., Material distribution in the partially filled zone of a twin-screw extruder. Advances in Polymer Technology, 18(4), 295-302, 1999.

Eitzlmayr, A., and Khinast, J., Co-rotating twin-screw extruders: Detailed analysis of conveying elements based on smoothed particle hydrodynamics. Part 1: Hydrodynamics. Chemical Engineering Science 134, 861-879, $2015 a$.

Eitzlmayr, A., \& Khinast, J., Co-rotating twin-screw extruders: Detailed analysis of conveying elements based on smoothed particle hydrodynamics. Part 2: Mixing. Chemical Engineering Science 134, 880-886, 2015 b.

Ellero, $\mathrm{M}$ and Tanner, R., Sph simulations of transient viscoelastic flows at low reynolds number. Journal of Non-Newtonian Fluid Mechanics, 132(1-3), 61-72, December 2005. 
Gómez-Gesteira, M., \& Dalrymple, R. A., Using a Three-Dimensional Smoothed Particle Hydrodynamics Method for Wave Impact on a Tall Structure. Journal of Waterway, Port, Coastal, and Ocean Engineering, 130(2), 63-69, 2004.

Ha, J., and Cleary, P.W., Comparison of SPH simulations of high pressure die casting with those of Schmidt and Klein, J. Cast Metals Res., 12, 409-418, 2000.

Hétu, J.-F., \& Ilinca, F., Immersed boundary finite elements for 3D flow simulations in twin-screw extruders. Computers \& Fluids, 87, 2-11, 2013.

Hosseini, S. M., Manzari, M. T., and Hannani, S. K., A fully explicit three-step sph algorithm for simulation of non-newtonian fluid flow. International Journal of Numerical Methods for Heat \& Fluid Flow, 17(7), 715-735, 2007.

Ishikawa, T., Amano, T., Kihara, S., and Funatsu, K., Flow patterns and mixing mechanisms in the screw mixing element of a co-rotating twin-screw extruder. Polymer Engineering \& Science, 42(5), 925-939, 2002.

Ishikawa, T., Kihara, S., and Funatsu, K., 3-d non-isothermal flow field analysis and mixing performance evaluation of kneading blocks in a co-rotating twin srew extruder. Polymer Engineering \& Science, 41(5), 840-849, 2001.

Kajiwara, T., Nagashima, Y., Nakano, Y., and Funatsu, K., Numerical study of twin-screw extruders by three-dimensional flow analysis-development of analysis technique and evaluation of mixing performance for full flight screws. Polymer Engineering \& Science, 36(16), 2142-2152, 1996. 
Kao, S. V. and Allison, G. R.. Residence time distribution in a twin screw extruder. Polymer Engineering and Science, 24(9), 645-651, 1984.

Kulasegaram, S., Karihaloo, B. L., \& Ghanbari, A., Modelling the flow of self-compacting concrete. International Journal for Numerical and Analytical Methods in Geomechanics, 35(6), 713-723, 2011.

Laigle, D., Lachamp, P., and Naaim, M., Sph-based numerical investigation of mudflow and other complex fluid flow interactions with structures. Computational Geosciences, 11(4), 297-306, December 2007.

Lawal, A. and Kalyon, D. M., Mechanisms of mixing in single and co-rotating twin screw extruders. Polymer Engineering \& Science, 35(17), 1325-1338, 1995.

Monaghan, J. J., Kos, A., and Issa, N., Fluid motion generated by impact. Journal of Waterway, Port, Coastal, and Ocean Engineering, 129(6), 250-259, 2003.

Monaghan, J. J., Simulating Free Surface Flows with SPH. Journal of Computational Physics, 110(2), 399-406, 1994.

Monaghan, J. J., Smoothed particle hydrodynamics. Reports on Progress in Physics, 68(8), 1703-1759, August 2005.

Rios, A. C., Gramann, P. J., and Osswald, T. A., Comparative study of mixing in corotating twin screw extruders using computer simulation. Advances in Polymer Technology, 17(2), 107-113, 1998. 
Robinson M, Cleary P, Monaghan J. Analysis of mixing in a twin-cam mixer using Smoothed Particle Hydrodynamics. AIChe Journal, 8(54), 1987-1998, 2008

Robinson, M. and Cleary, P. W., The influence of cam geometry and operating conditions on chaotic mixing of viscous fluids in a twin cam mixer. AIChE Journal, 57, 581-598, 2011.

Robinson, M., \& Cleary, P. W., Flow and mixing performance in helical ribbon mixers. Chemical Engineering Science, 84, 382-398, 2012.

Rodriguez-Paz, M.X. and Bonet, J., A corrected smooth particle hydrodynamics method for the simulation of debris flows. Numerical Methods for Partial Differential Equations, 20(1), 140-163, 2004.

Shadden SC, Lekien F, Marsden JE. Definition and properties of Lagrangian coherent structures from finite-time Lyapunov exponents in two-dimensional aperiodic flows. Physica D Nonlinear Phenomena. 212, 271-304, 2005.

Shamekhi, A., Sadeghy, K., Bahrami, M. N., and Naei, M. H., Using mesh free method for numerical simulation of non-newtonian fluid flow over a step. Journal of the Society of Rheology, Japan, 36(1), 19-27, 2008.

Shao, S. and Lo, E., Incompressible sph method for simulating newtonian and nonnewtonian flows with a free surface. Advances in Water Resources, 26(7), 787-800, 2003.

Sobhani, H., Anderson, P. D., Meijer, H. H. E., Ghoreishy, M. H. R., \& Razavi-Nouri, M.,. Non-Isothermal Modeling of a Non-Newtonian Fluid Flow in a Twin Screw Extruder Using the Fictitious Domain Method. Macromolecular Theory and Simulations, 22(9), 462-474, 
2013.

Valette, R., Vergnes, B., and Coupez, T., Multiscale simulation of mixing processes using 3d-parallel, fluid-structure interaction techniques. International Journal of Material Forming, 1, 1131-1134, 2008.

Van Der Goot, A. J., Pooiwer, O., and Janssen, L. P. B. M., Determination of the degree of fill in a counter-rotating twin screw extruder. Polymer Engineering and Science, 38(7), 1193-1198, 1998.

Van Der Goot, A. J., Poorter, O., and Janssen, L. P. B. M., Determination of the degree of fill in a counter-rotating twin screw extruder. Polymer Engineering \& Science, 38(7), 11931198, 1998.

Veen, D., \& Gourlay, T., A combined strip theory and Smoothed Particle Hydrodynamics approach for estimating slamming loads on a ship in head seas. Ocean Engineering, 43, 64$71,2012$.

Wittek, P., Pereira, G. G., Emin, A., and Cleary, P. W., Analysis of flow in an extruder with a kneading element using SPH, Chem. Eng. Sci., 187, 256-268, 2018.

Yakutovich, M. V., Newton, C. J. P., \& Cleaver, D. J., Mesh-Free Simulation of Complex LCD Geometries. Molecular Crystals and Liquid Crystals, 502(1), 245-257, 2009. 


\section{Figures}

a)

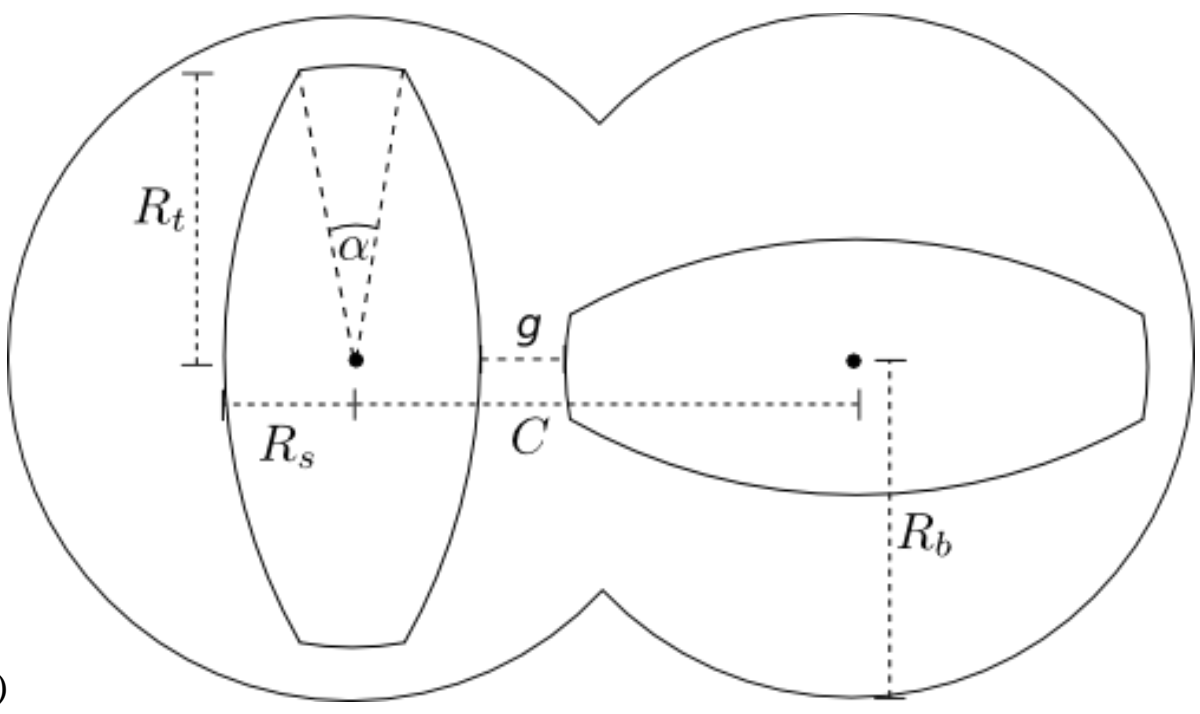

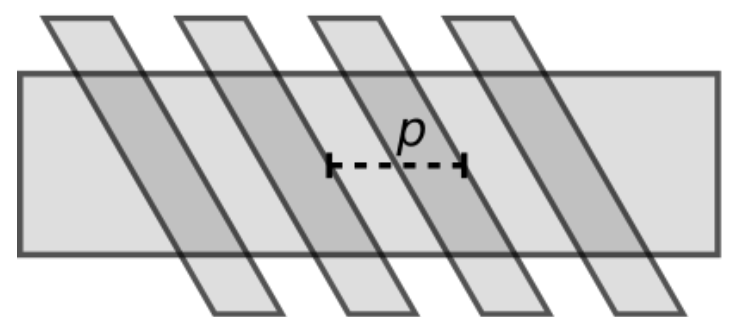

b)

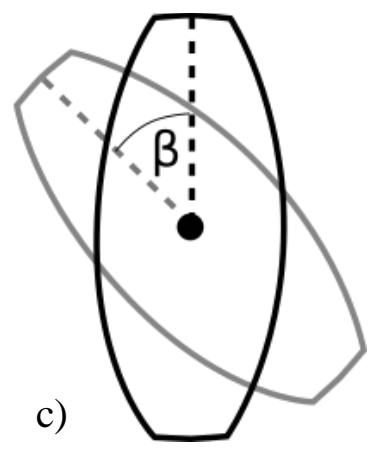

Figure 1: a) Cross-section of the Twin screw Extruder. $R_{t}=25 \mathrm{~mm}$ and $R_{s}=13.75 \mathrm{~mm}$ is the maximum and minimum radii of the impeller cross-section. The centres of each barrel (and each screw element) are separated by $C=40.25 \mathrm{~mm}$, and the radius of each barrel is $\mathrm{R}_{b}=26.5 \mathrm{~mm}$. The angle of each screw tip is $\alpha=$ 11.61 degrees. The minimum spacing between the elements of the opposite impeller $g$, is set by the separation of the two barrels $C$. b) Diagram showing a schematic side view of a screw impellor. The screw pitch length $p$ is the distance between adjacent 180 degree sweeps of the screw tips along the axis of the impellor. c) Diagram showing the view along the rotation axis of a kneading block elements. The kneading block stagger angle $\beta$ is the angle of rotation between subsequent discrete blocks. 


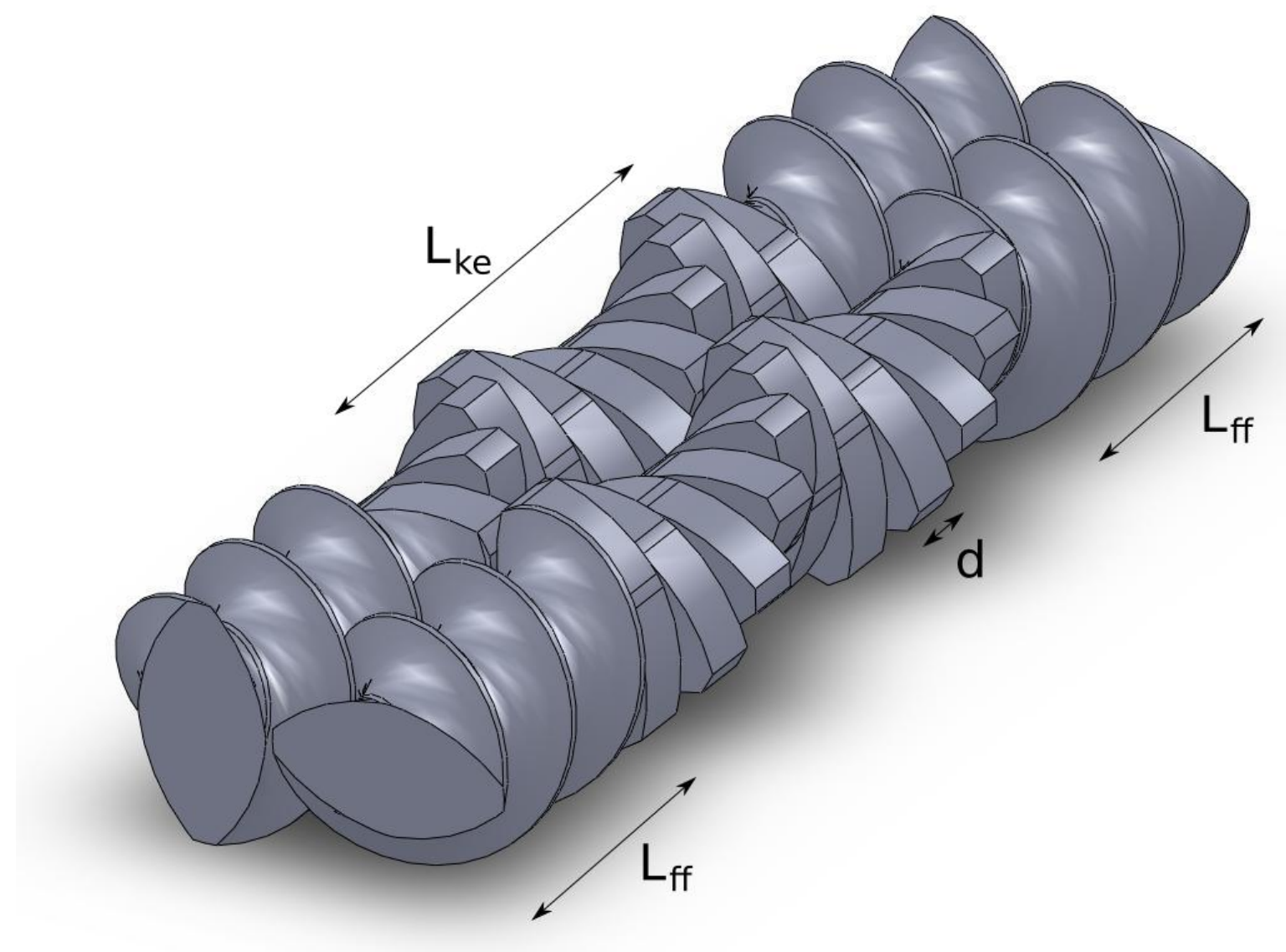

Figure 2: Impeller design with screw elements on either side of a set of kneading blocks. The outer casing of the mixer is not shown. Key geometric lengths are shown on the diagram. 


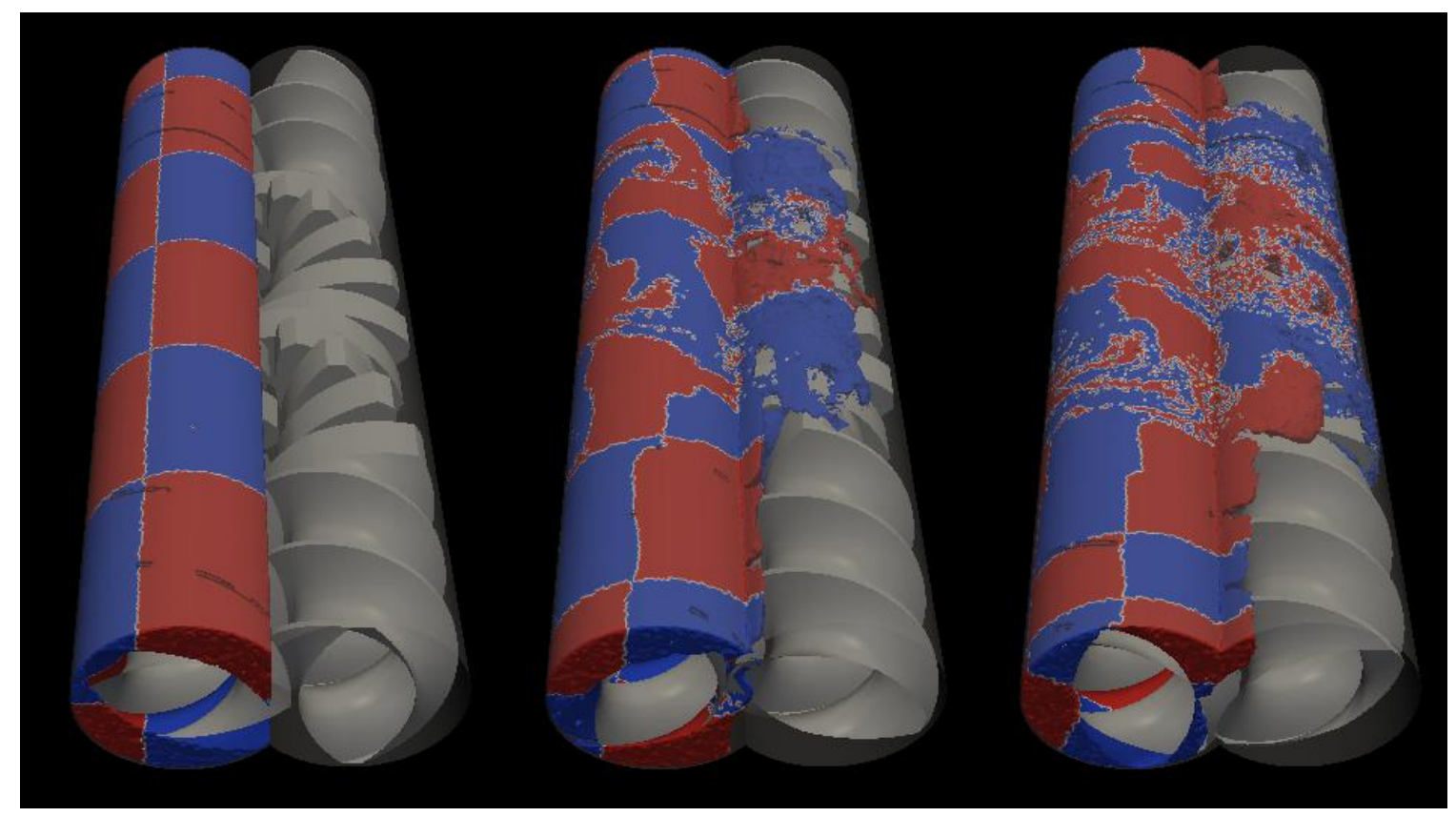

Figure 3: Cross-channel mixing visualisation. The fill level in the mixer is $100 \%$, and his figure shows only the evolution of Volume One for $\mathrm{t}=0$ (left), $\mathrm{t}=2 \mathrm{sec}$ (middle) and $\mathrm{t}=4 \mathrm{sec}$ (right).

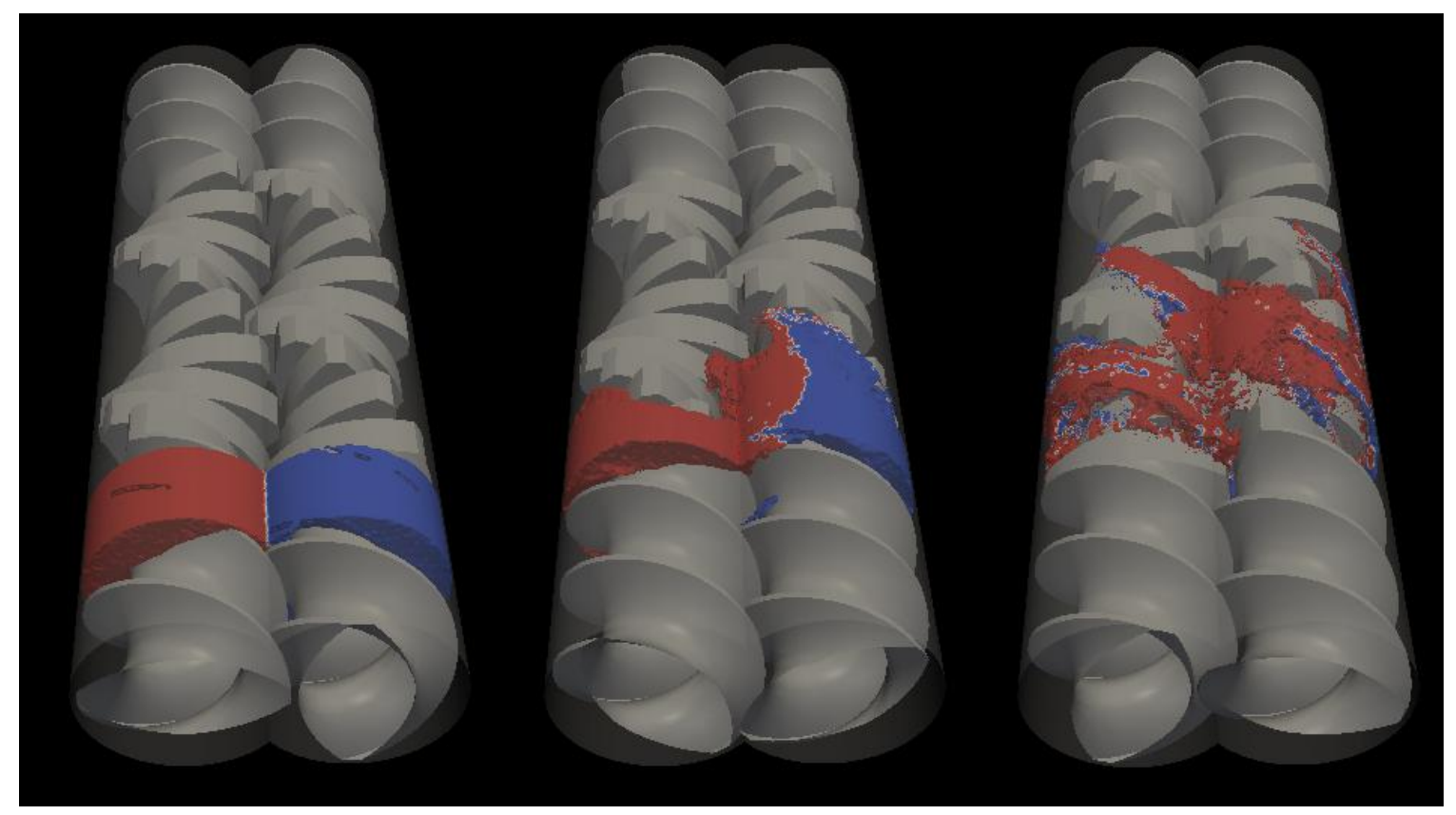

Figure 4: Axial mixing visualisation. The fill level of the mixer is $100 \%$, and this figure shows only the evolution of Volume Two for $t=0 \mathrm{sec}$ (left), $t=2 \mathrm{sec}$ (middle) and $t=4 \mathrm{sec}$ (right). 


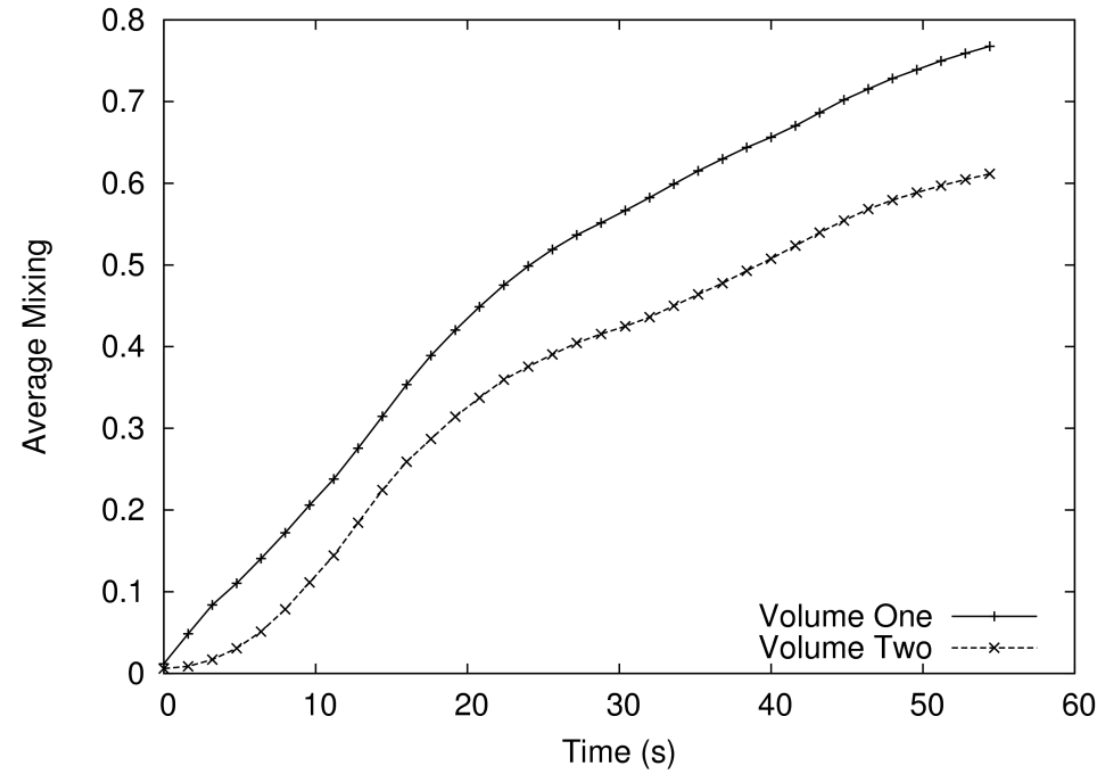

Figure 5: Variation of the average mixing measure $\langle\mathrm{M}\rangle$ with time for Volume One (cross-channel mixing) and Volume Two (axial mixing) 


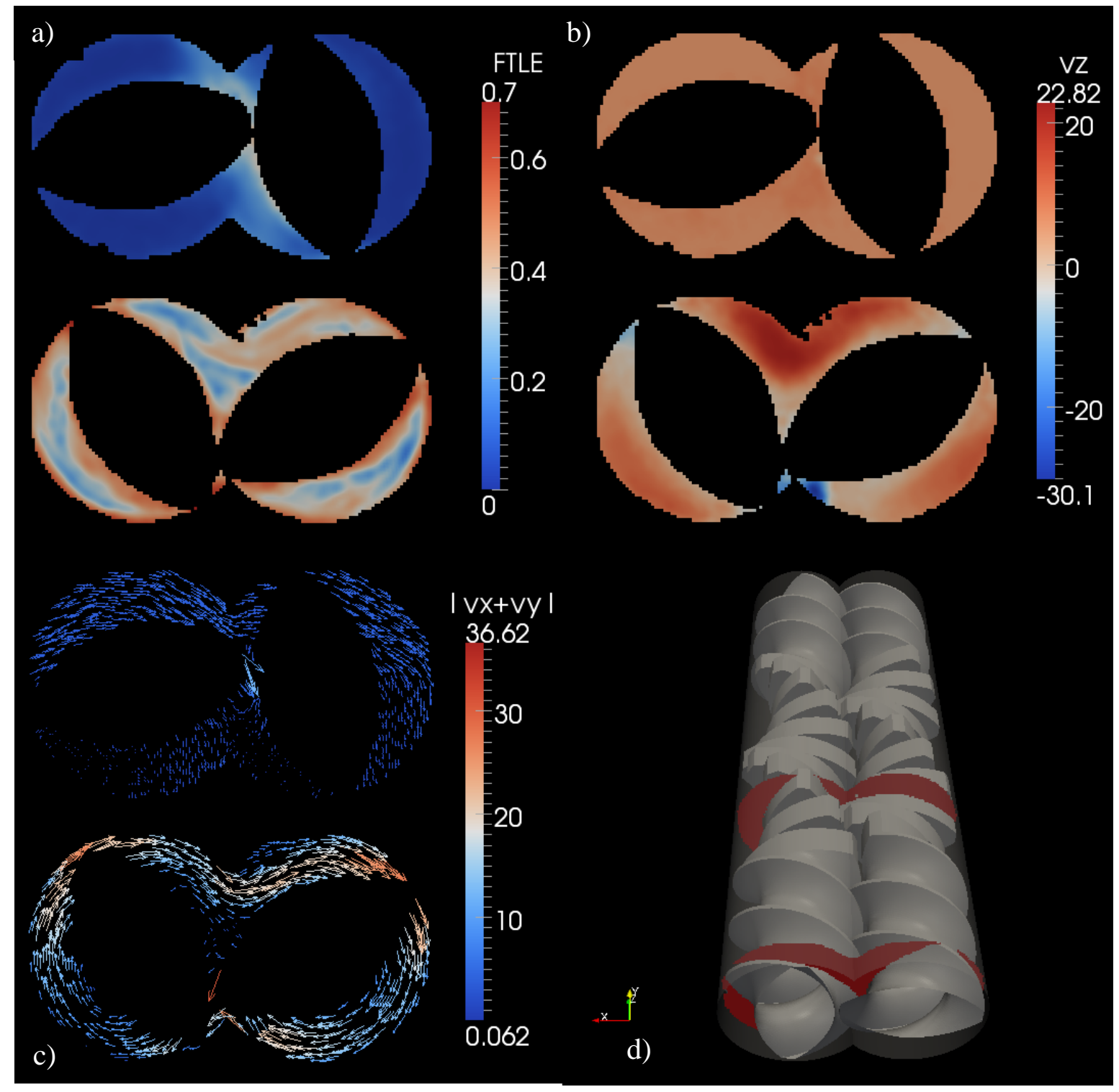

Figure 6: Flow and mixing structure for an impeller with screw and kneading blocks elements, a) FTLE distribution in cross-section of the screw (top cross-section in each pair) and the kneading blocks (bottom cross-section of the pair), b) axial flow speed in the screw and kneading block cross-sections, c) flow velocity in each axial section with direction shown by the arrows and speed given by the colour and arrow length, d) view of the full impeller with the positions of screw and kneading block cross-sections shown as red planes. Velocities are given in units of mm/s. 


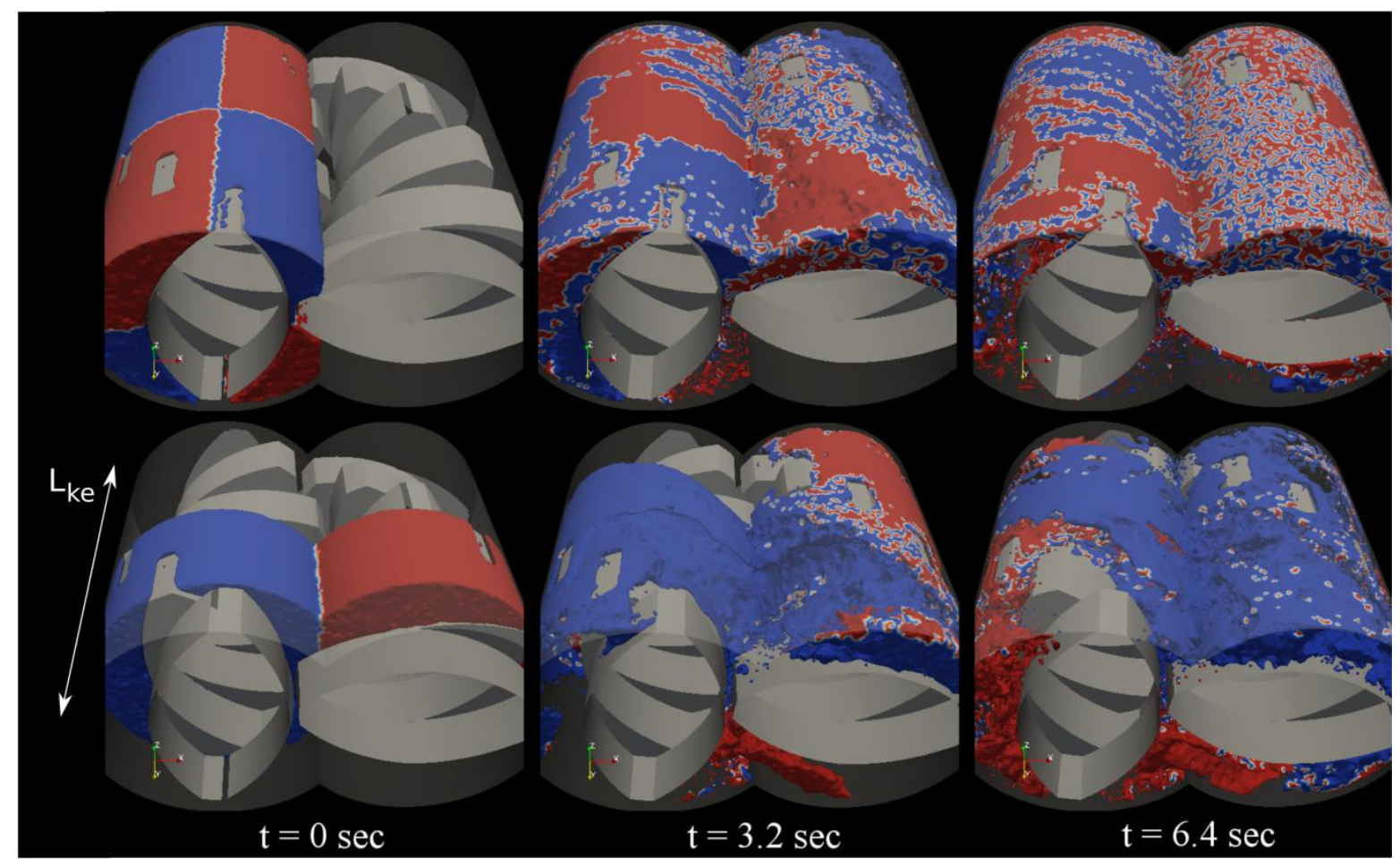

Figure 7: Time sequence showing the transport of Volume One and Two using the kneading blocks geometry.

Top and bottom rows show Volume One and Two which demonstrate cross-chamber and axial mixing respectively. 


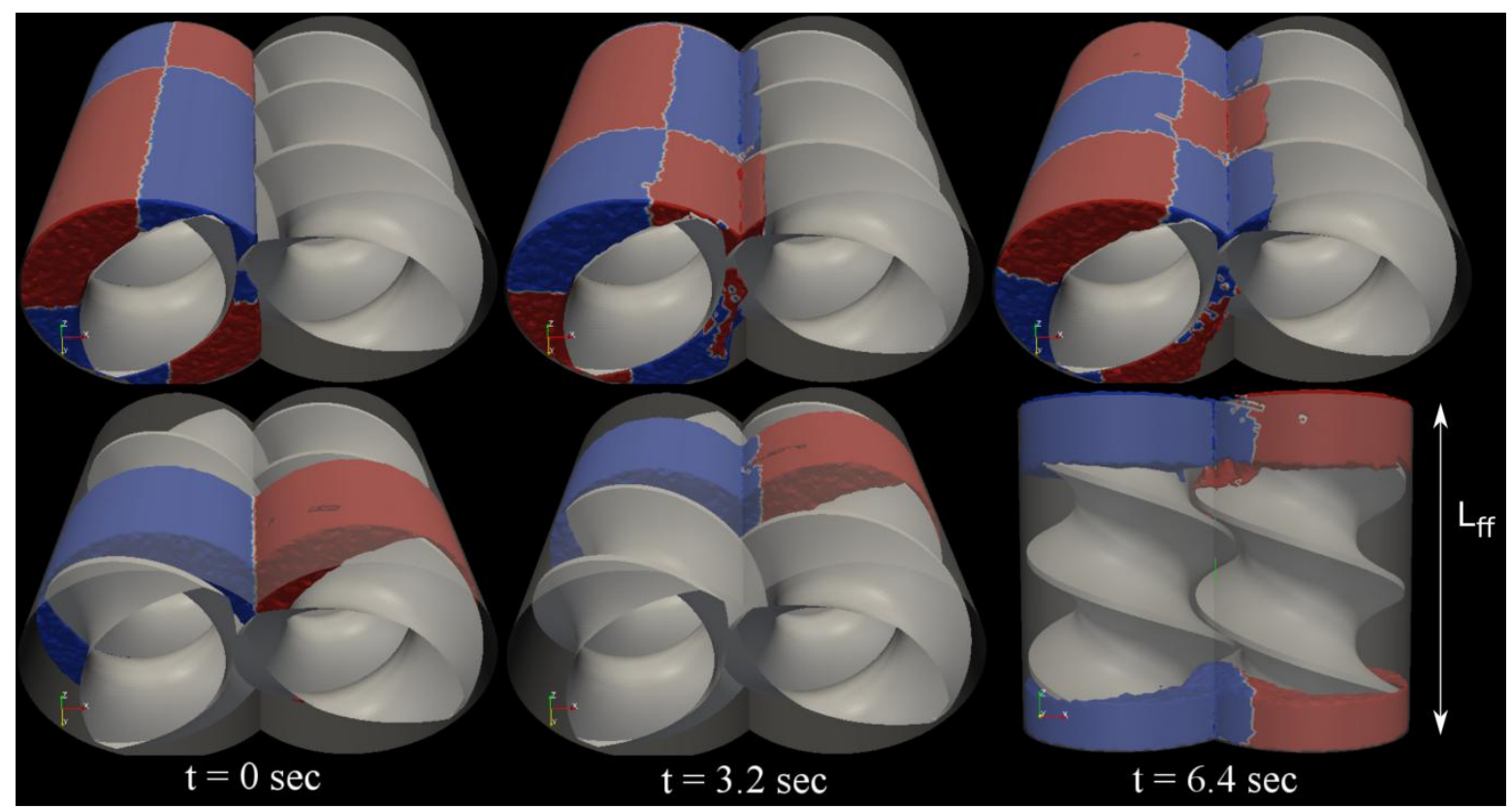

Figure 8: Time sequence showing the transport of Volume One (top row) and Volume Two (bottom row) using the screw elements which demonstrate cross-chamber and axial mixing respectively. 

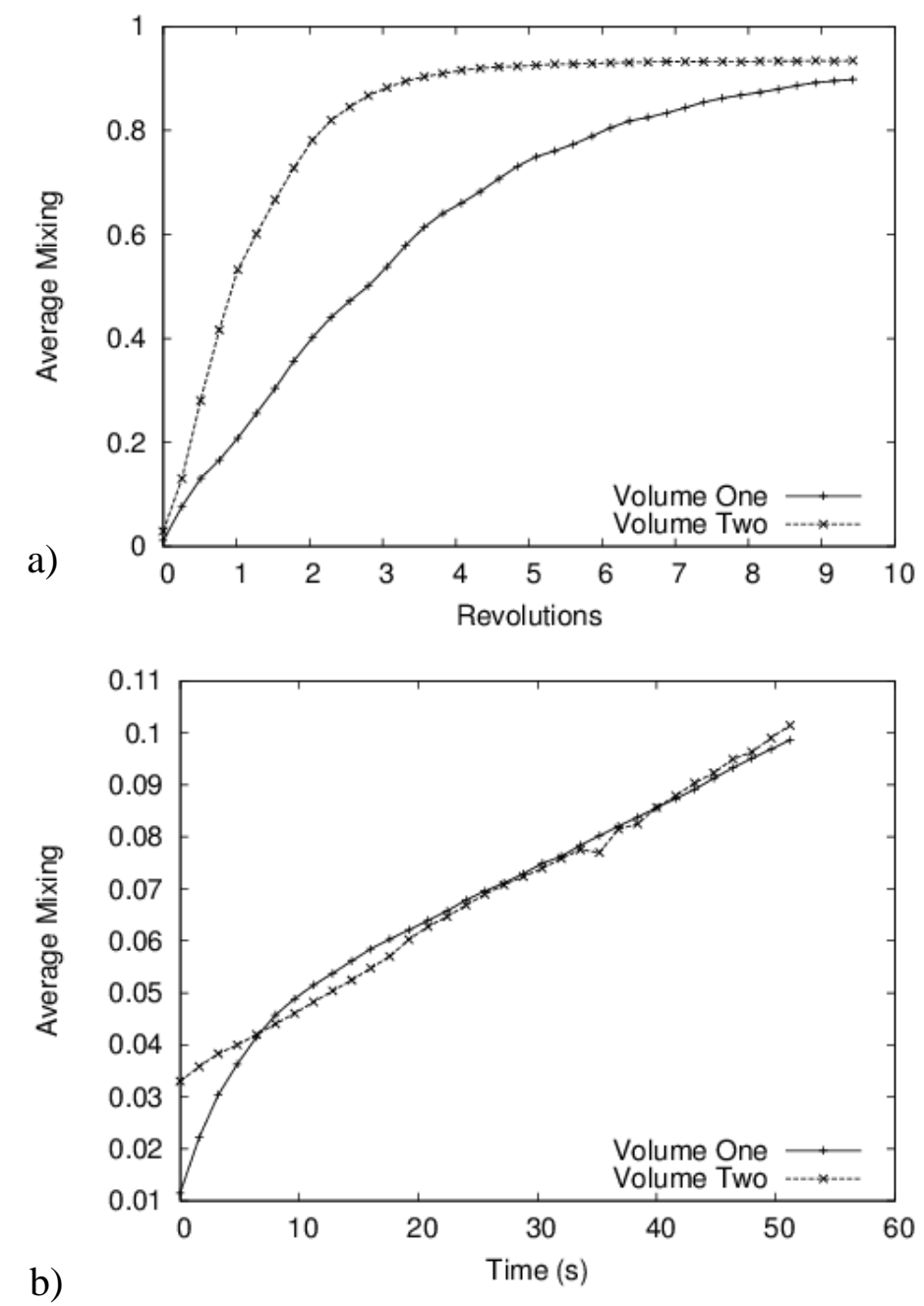

Figure 9: Variation of the average mixing measure with time for the, a) isolated kneading blocks, and b) screw elements. Each plot shows the mixing of both representative volumes over 9.5 revolutions of the impellors. The tick labels on the time axis are given in seconds (bottom) and number of revolutions (top). Volume 1 measures cross-chamber mixing while Volume 2 measures axial mixing. 

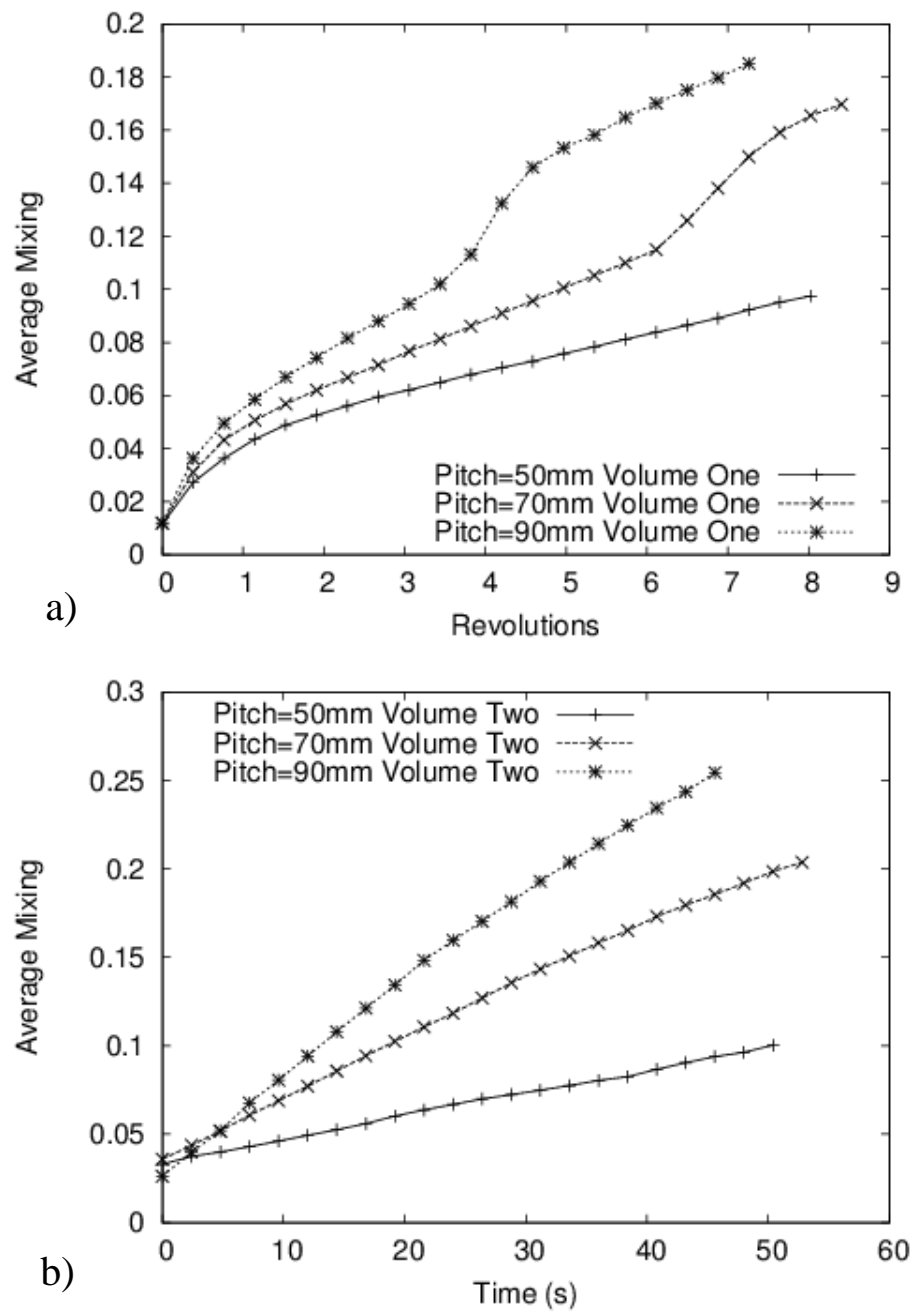

Figure 10: Variation of the average mixing measure with time for the screw elements with varying pitch $p$, a) for Volume 1 reflecting cross-chamber mixing, and b) for Volume 2 reflecting axial mixing. 

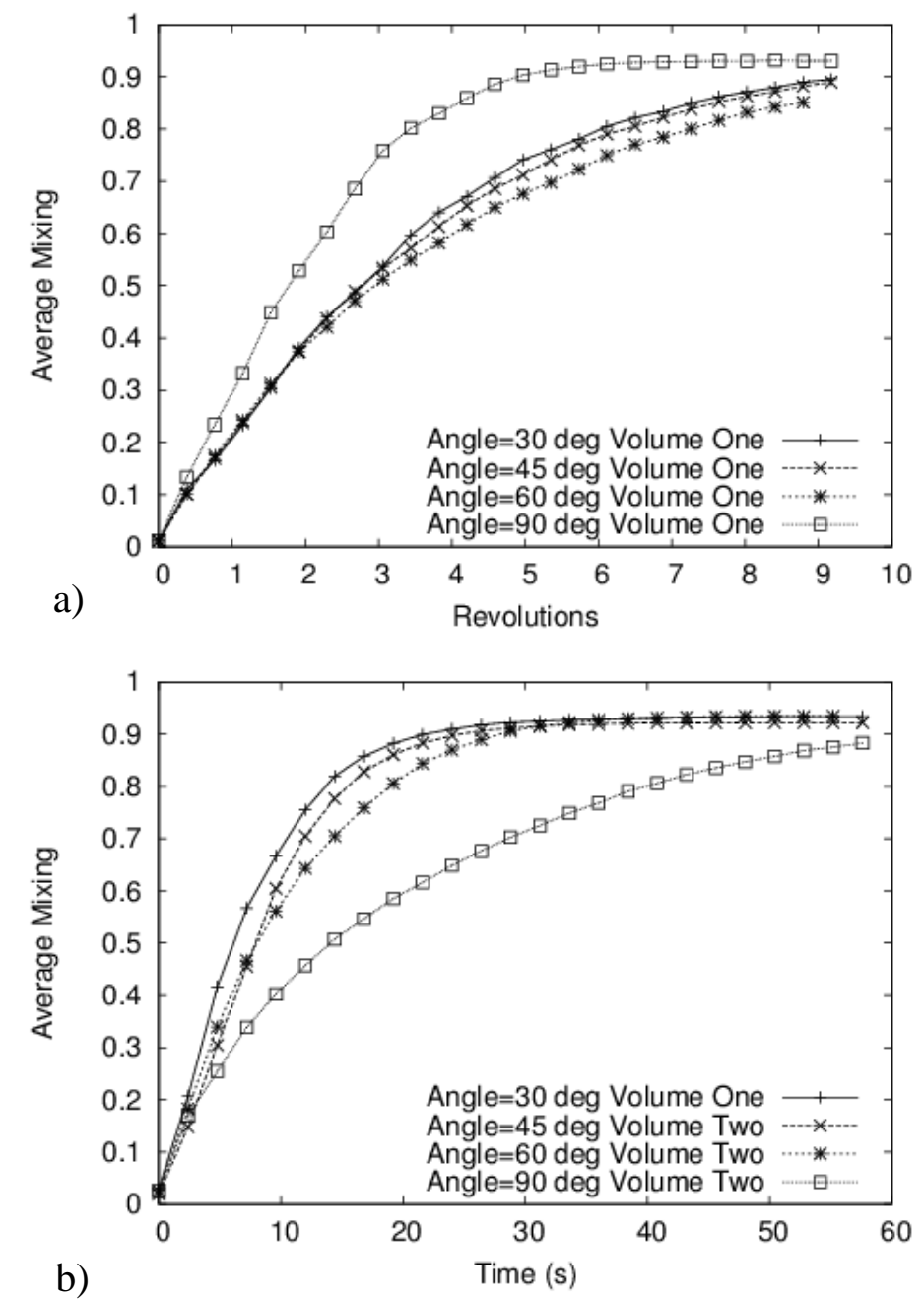

Figure 11: Variation of the average mixing measure with time for the kneading block impellors with varying block stagger angle $\beta$, a) for Volume 1 reflecting cross-chamber mixing, and b) for Volume 2 reflecting axial mixing. 

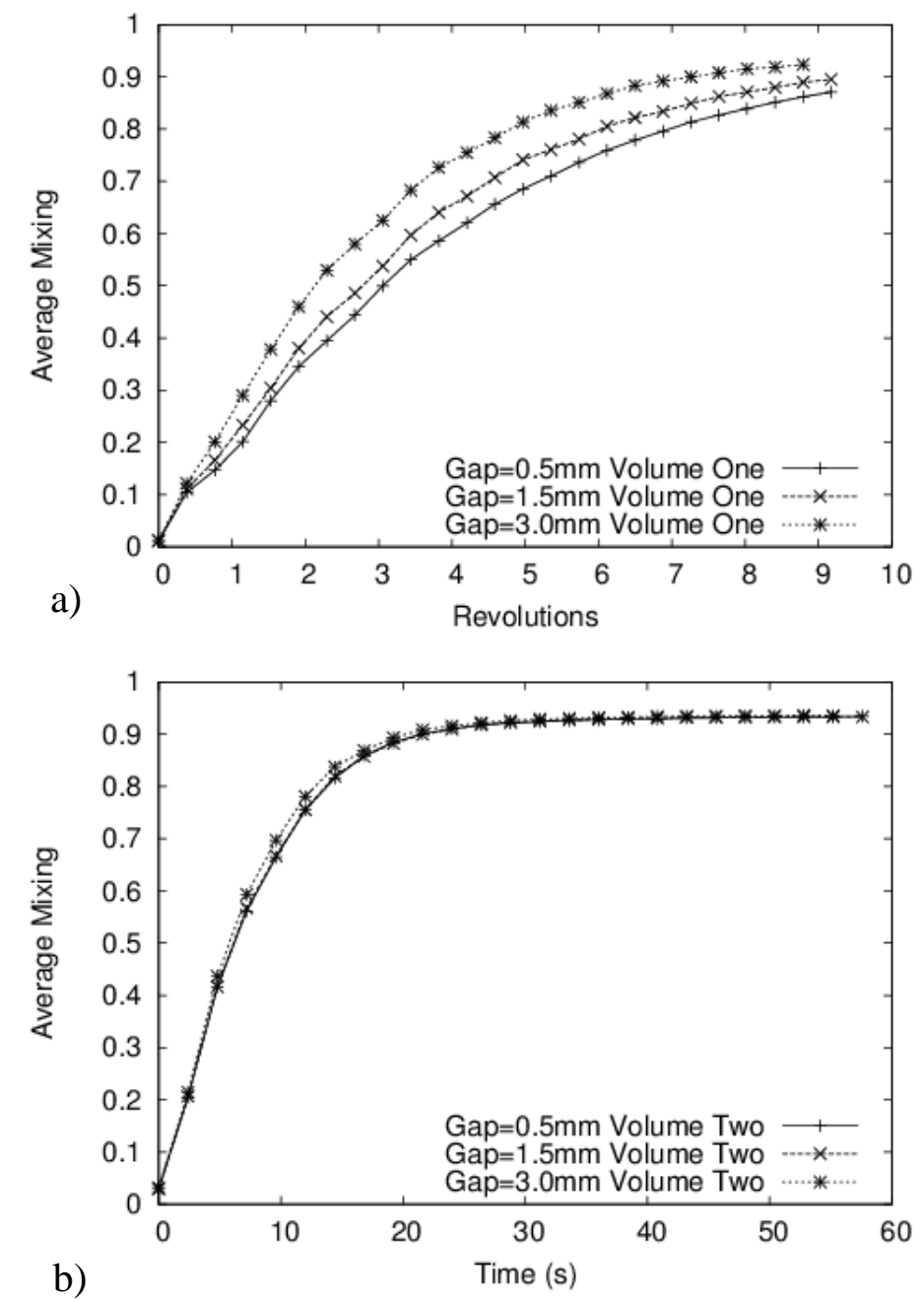

Figure 12: Variation of the average mixing measure with time for the kneading blocks with varying gap width $g$, a) for Volume 1 reflecting cross-chamber mixing, and b) for Volume 2 reflecting axial mixing. 

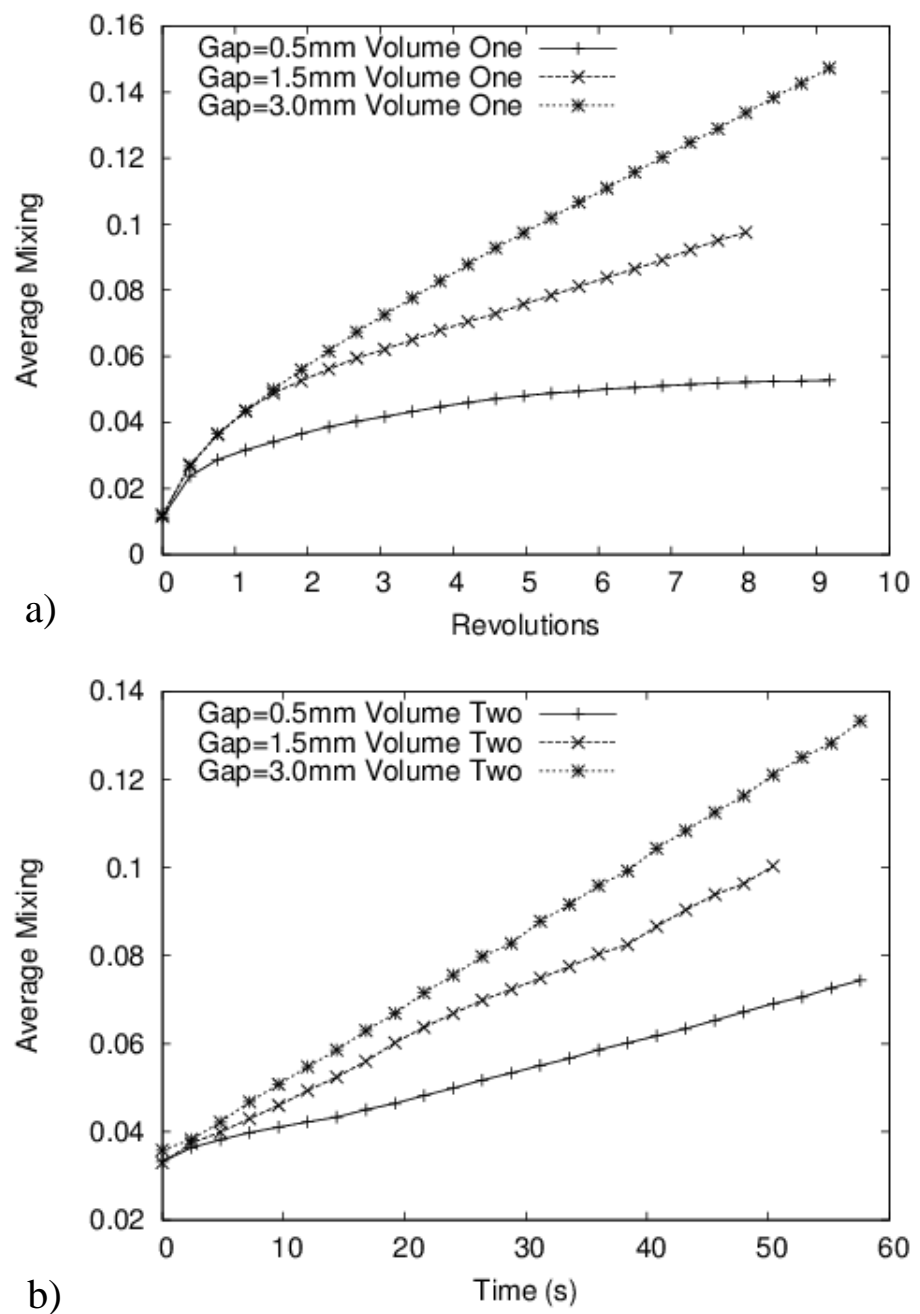

Figure 13: Variation of the average mixing measure with time for the screw elements with varying gap width $g$, a) for Volume 1 reflecting cross-chamber mixing, and b) for Volume 2 reflecting axial mixing. 


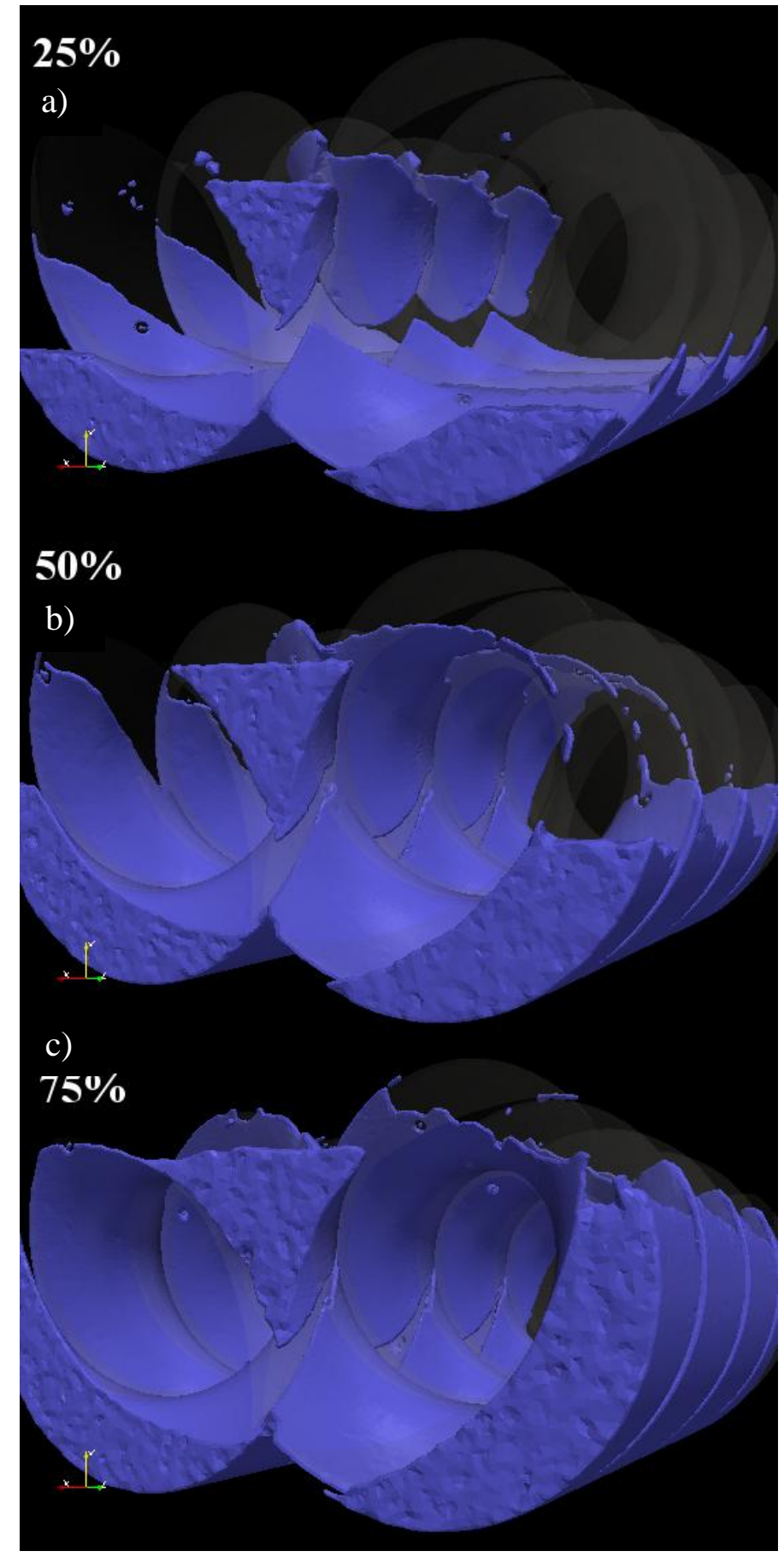

Figure 14: Free surface of water around screw elements with varying fill percentage, a) $25 \%$ volumetric fill, b) $50 \%$ fill and b) $75 \%$ fill. 

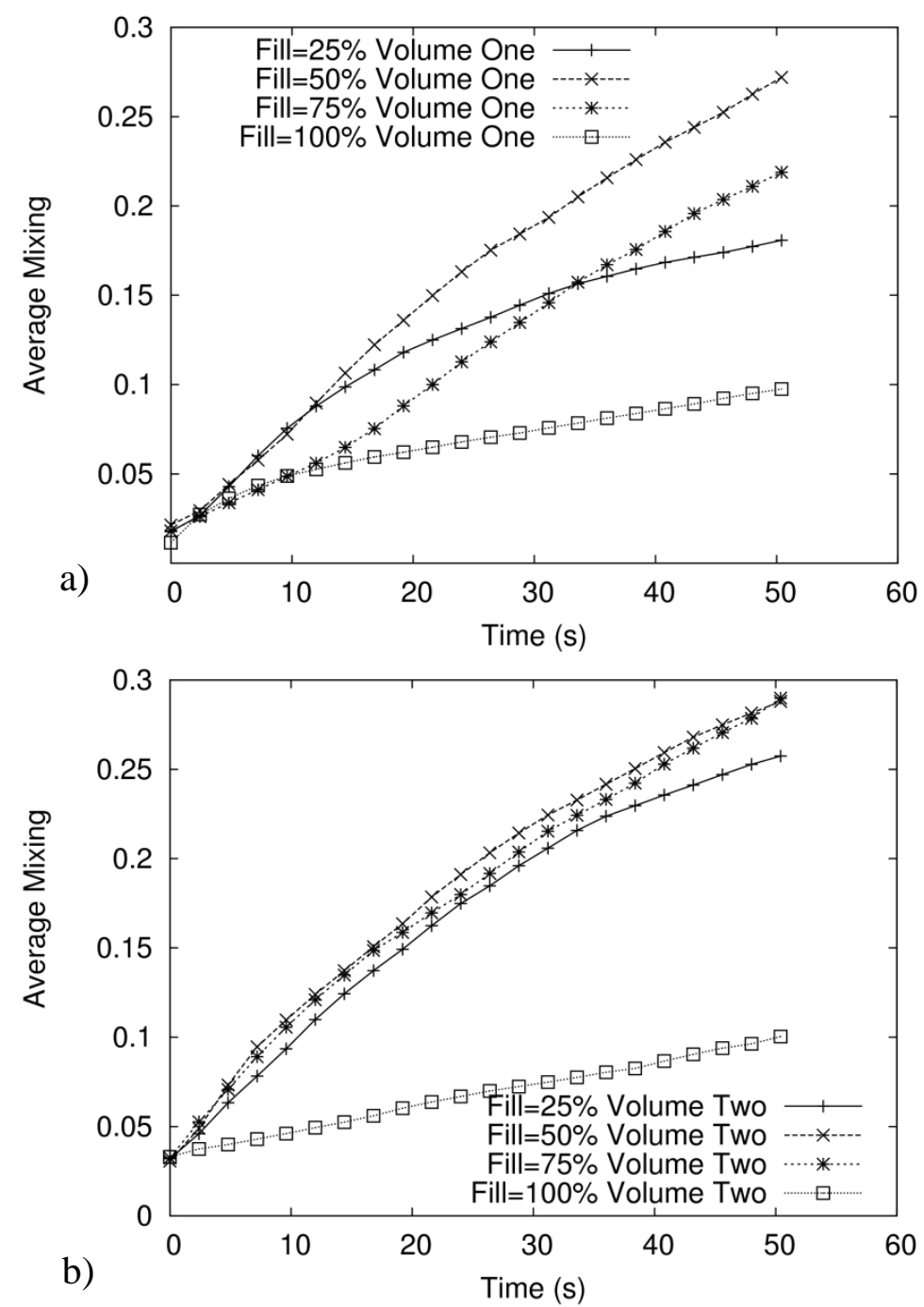

Figure 15: Variation of the average mixing measure with time for the screw impellors with varying fill percentage, a) for Volume 1 reflecting cross-chamber mixing, and b) for Volume 2 reflecting axial mixing. 


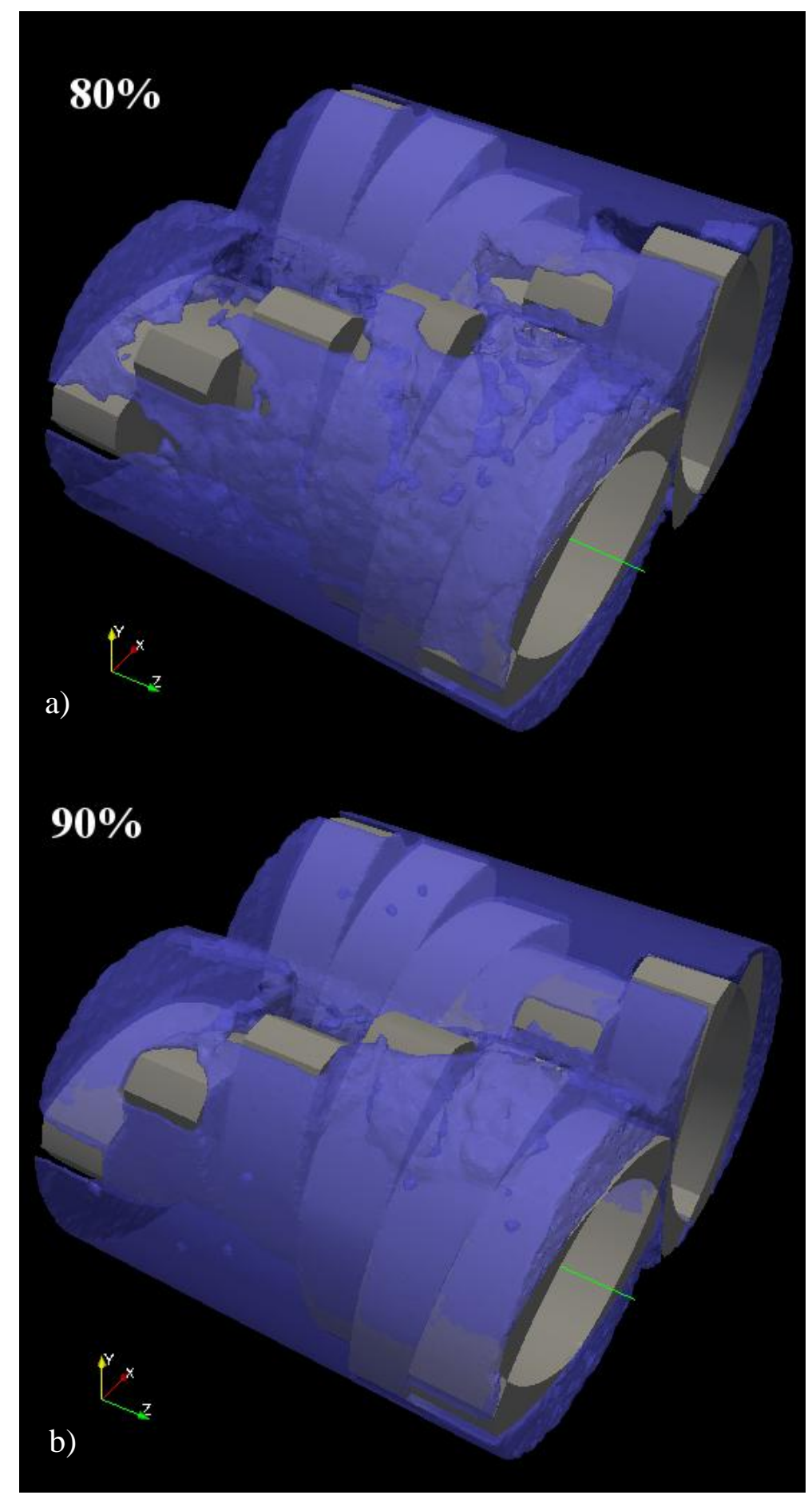

Figure 16: Free surface of fluid around kneading blocks with varying fill percentage, a) $80 \%$ volumetric fill, and b) $90 \%$ fill. 

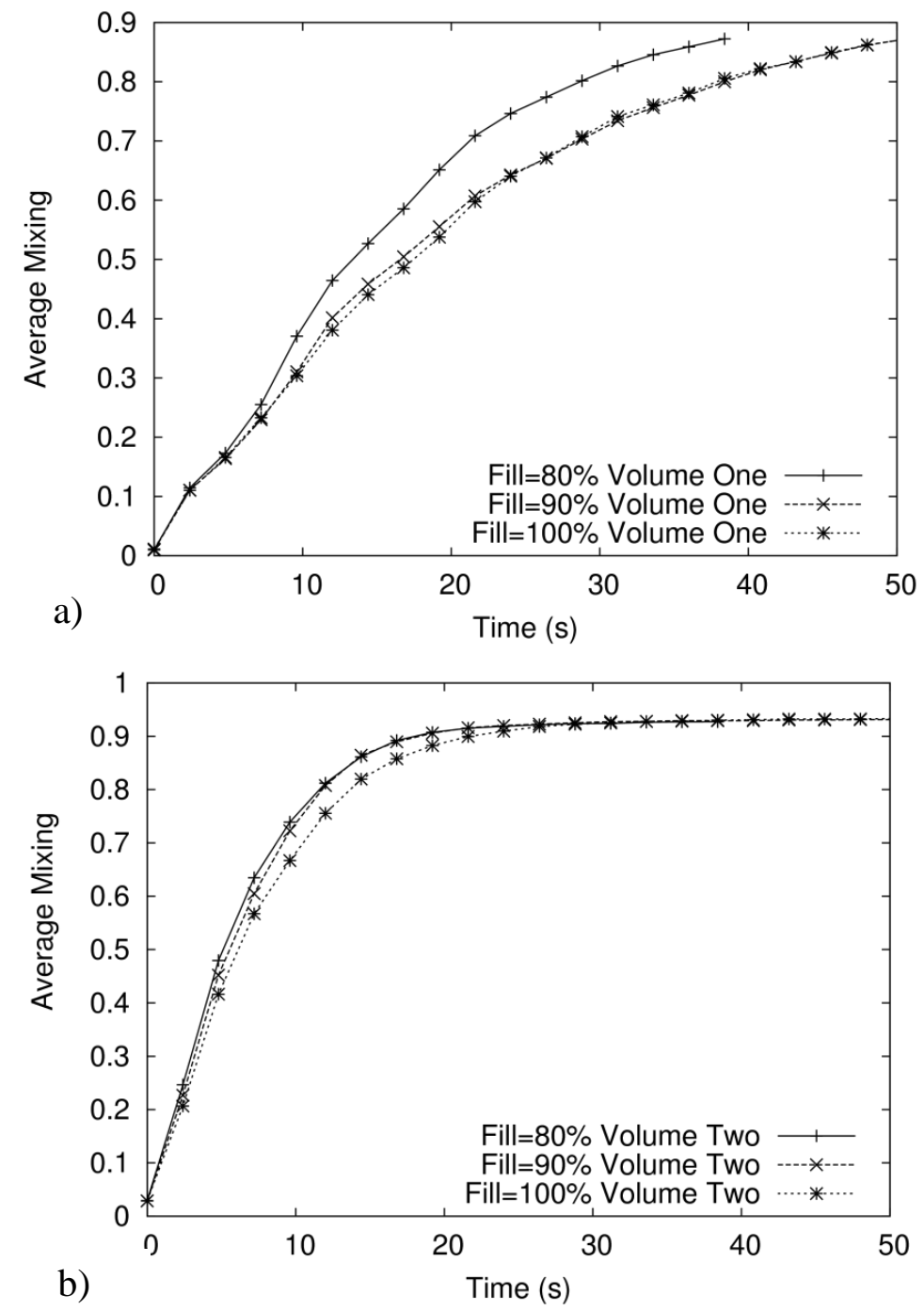

Figure 17: Variation of the average mixing measure with time for the kneading blocks with varying fill percentage, a) for Volume 1 reflecting cross-chamber mixing, and b) for Volume 2 reflecting axial mixing. 


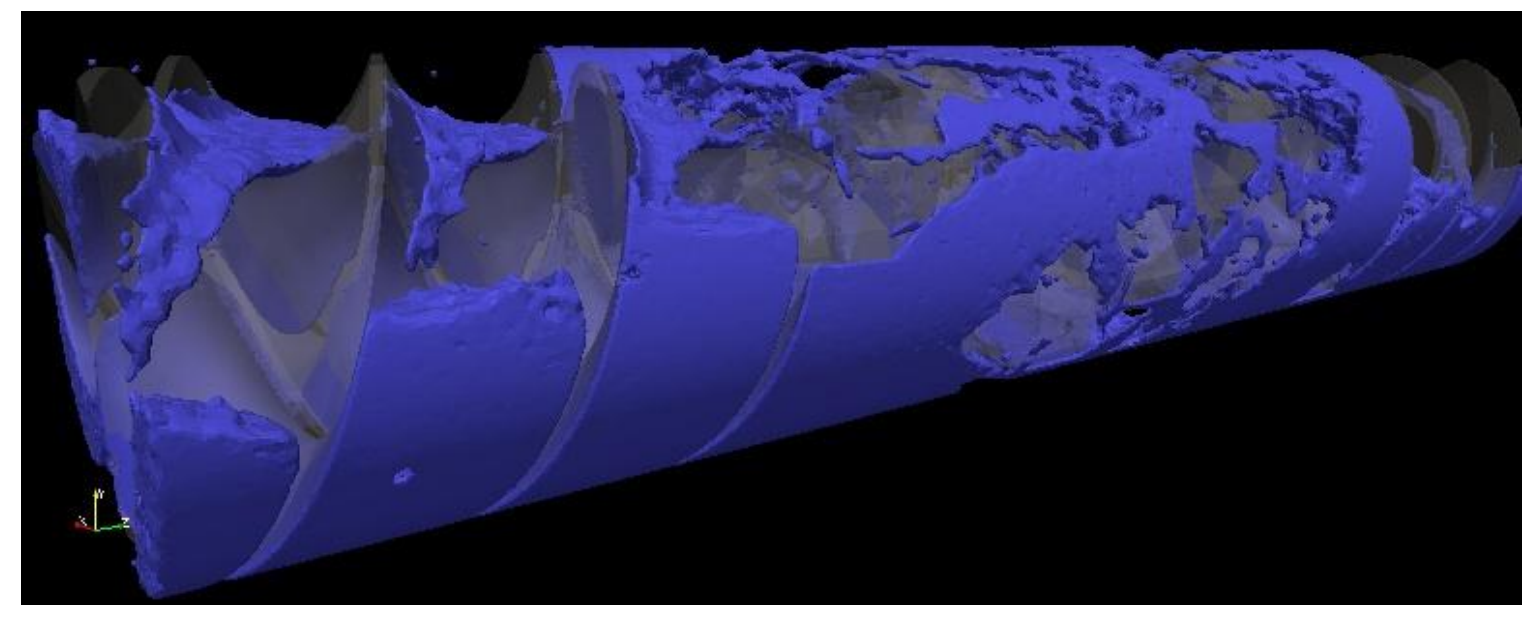

Figure 18: Free surface for partially filled (50\%) combined extruder geometry. The pitch of the kneading blocks is the same (30 degrees) as the screw elements.

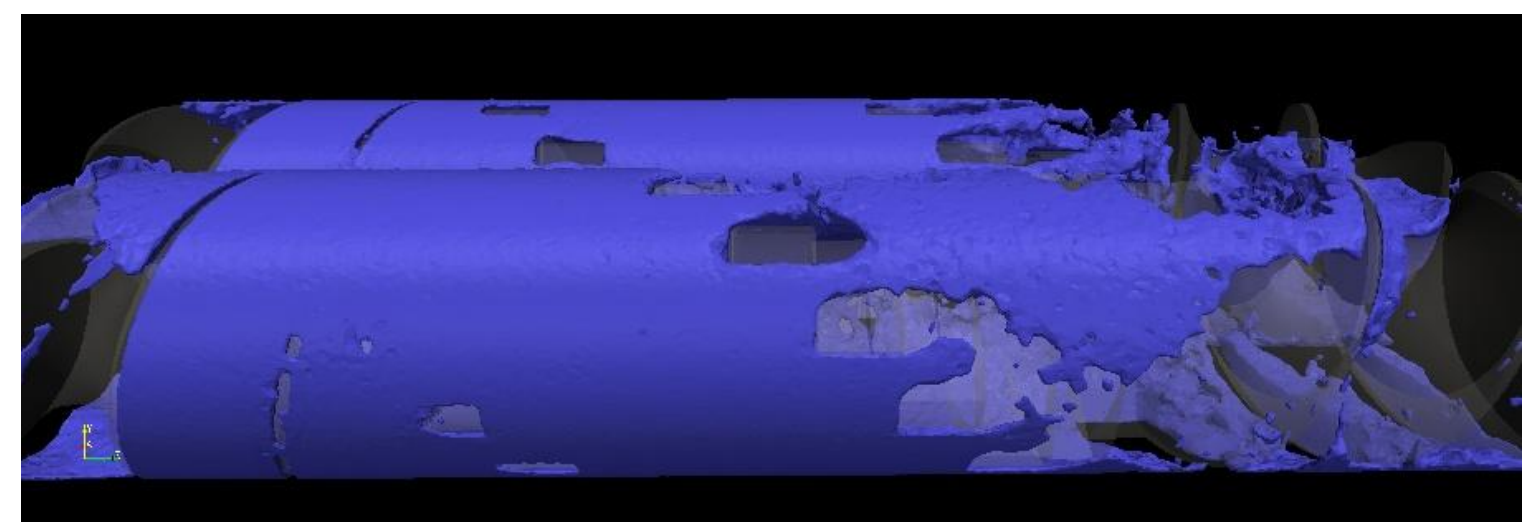

Figure 19: Free surface for partially filled (50\%) combined extruder geometry. The pitch of the kneading blocks is opposite (-30 degrees) to the screw elements, resulting in a build-up of fluid in the kneading block mixing zone. 

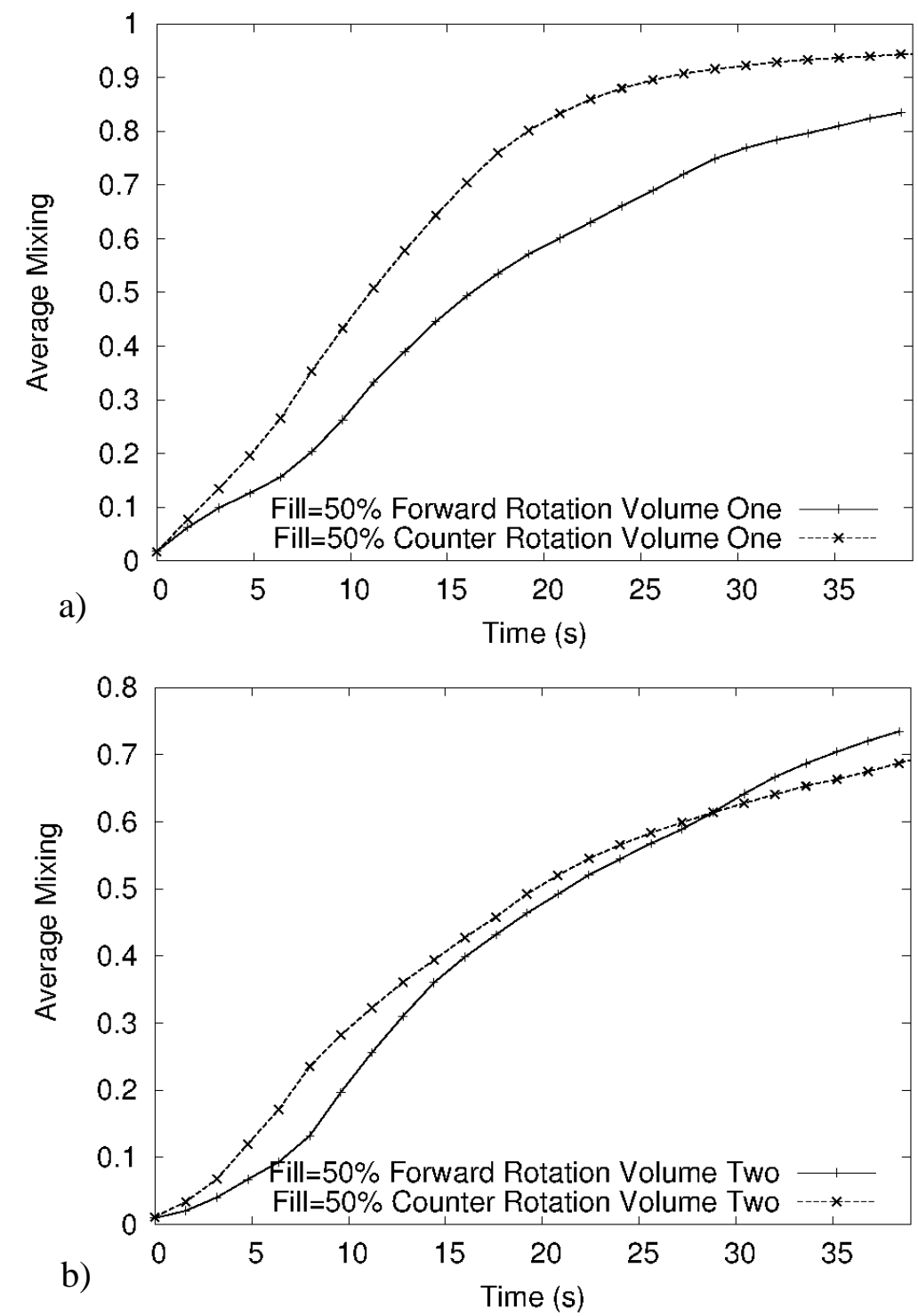

Figure 20: Variation of the average mixing measure with time for the combined kneading and screw geometry with 50\% fill level, a) for Volume 1 reflecting cross-chamber mixing, and b) for Volume 2 reflecting axial mixing. 